Article

\title{
Predicting Maximal Gaps in Sets of Primes
}

\author{
Alexei Kourbatov ${ }^{1, *,+}$ and Marek Wolf $2, *,+$ \\ 1 JavaScripter.net, 15127 NE 24th St., \#578, Redmond, WA 98052, USA \\ 2 Faculty of Mathematics and Natural Sciences, Cardinal Stefan Wyszynski University, \\ Wóycickiego 1/3, Bldg. 21, PL-01-938 Warsaw, Poland \\ * Correspondence: akourbatov@gmail.com (A.K.); m.wolf@uksw.edu.pl (M.W.) \\ + The authors contributed equally to this work.
}

Received: 5 April 2019; Accepted: 29 April 2019; Published: 4 May 2019

check for updates

\begin{abstract}
Let $q>r \geq 1$ be coprime integers. Let $\mathbb{P}_{c}=\mathbb{P}_{c}(q, r, \mathcal{H})$ be an increasing sequence of primes $p$ satisfying two conditions: (i) $p \equiv r(\bmod q)$ and (ii) $p$ starts a prime $k$-tuple with a given pattern $\mathcal{H}$. Let $\pi_{c}(x)$ be the number of primes in $\mathbb{P}_{c}$ not exceeding $x$. We heuristically derive formulas predicting the growth trend of the maximal gap $G_{\mathcal{C}}(x)=\max _{p^{\prime} \leq x}\left(p^{\prime}-p\right)$ between successive primes $p, p^{\prime} \in \mathbb{P}_{c}$. Extensive computations for primes up to $10^{14}$ show that a simple trend formula $G_{c}(x) \sim \frac{x}{\pi_{c}(x)} \cdot\left(\log \pi_{c}(x)+O_{k}(1)\right)$ works well for maximal gaps between initial primes of $k$-tuples with $k \geq 2$ (e.g., twin primes, prime triplets, etc.) in residue class $r(\bmod q)$. For $k=1$, however, a more sophisticated formula $G_{c}(x) \sim \frac{x}{\pi_{c}(x)} \cdot\left(\log \frac{\pi_{c}^{2}(x)}{x}+O(\log q)\right)$ gives a better prediction of maximal gap sizes. The latter includes the important special case of maximal gaps in the sequence of all primes $(k=1, q=2, r=1)$. The distribution of appropriately rescaled maximal gaps $G_{c}(x)$ is close to the Gumbel extreme value distribution. Computations suggest that almost all maximal gaps satisfy a generalized strong form of Cramér's conjecture. We also conjecture that the number of maximal gaps between primes in $\mathbb{P}_{c}$ below $x$ is $O_{k}(\log x)$.
\end{abstract}

Keywords: Cramér conjecture; Gumbel distribution; prime gap; prime $k$-tuple; residue class; Shanks conjecture; totient

MSC: 11A41, 11N05

\section{Introduction}

A prime gap is the difference between consecutive prime numbers. The sequence of prime gaps behaves quite erratically (see OEIS A001223 [1]). While the prime number theorem tells us that the average gap between primes near $x$ is about $\log x$, the actual gaps near $x$ can be significantly larger or smaller than $\log x$. We call a gap maximal if it is strictly greater than all gaps before it. Large gaps between primes have been studied by many authors; see, e.g., [2-11]. In the early 1910s, Ramanujan considered maximal prime gaps up to low 7-digit primes [2], p. 133. More than a century later, we know all maximal gaps between primes below $2^{64}$ [3].

Let $G(x)$ be the maximal gap between primes not exceeding $x$ :

$$
G(x)=\max _{p_{n+1} \leq x}\left(p_{n+1}-p_{n}\right)
$$

Estimating $G(x)$ is a subtle and delicate problem. Cramér [4] conjectured on probabilistic grounds that $G(x)=O\left(\log ^{2} x\right)$, while Shanks [5] heuristically found that $G(x) \sim \log ^{2} x$. Granville [6] heuristically argued that for a certain subsequence of maximal gaps we should expect significantly larger sizes of $G(x)$; namely, $\limsup _{x \rightarrow \infty} \frac{G(x)}{\log ^{2} x} \geq 2 e^{-\gamma} \approx 1.1229$. 
Baker, Harman, and Pintz [7] proved that $G(x)=O\left(x^{0.525}\right)$; indeed, computation suggests that $G(x)<x^{0.525}$ for $x \geq 127$. Ford, Green, Konyagin, Maynard, and Tao [8] proved that the order of $G(x)$ is at least $\frac{c \log x \log \log x \log \log \log \log x}{\log \log \log x}$, solving a long-standing conjecture of Erdős.

Earlier, we independently proposed formulas closely related to the Cramér and Shanks conjectures. Wolf [12-14] expressed the probable size of maximal gaps $G(x)$ in terms of the prime-counting function $\pi(x)$ :

$$
G(x) \sim \frac{x}{\pi(x)} \cdot\left(\log \frac{\pi^{2}(x)}{x}+O(1)\right)
$$

which suggests an analog of Shanks conjecture $G(x) \sim \log ^{2} x-2 \log x \log \log x+O(\log x)$; see also Cadwell [15]. Extending the problem statement to prime $k$-tuples, Kourbatov [16,17] empirically tested (for $x \leq 10^{15}, k \leq 7$ ) the following heuristic formula for the probable size of maximal gaps $G_{k}(x)$ between prime $k$-tuples below $x$ :

$$
G_{k}(x) \sim a(x) \cdot\left(\log \frac{x}{a(x)}+O(1)\right)
$$

where $a(x)$ is the expected average gap between the particular prime $k$-tuples near $x$. Similar to (1), Formula (2) also suggests an analog of the Shanks conjecture, $G_{k}(x) \sim C \log ^{k+1} x$, with a negative correction term of size $O_{k}\left((\log x)^{k} \log \log x\right)$; see also [18,19].

In this paper we study a further generalization of the prime gap growth problem, viz.: What happens to maximal gaps if we only look at primes in a specific residue class mod $q$ ? The new problem statement subsumes, as special cases, maximal prime gaps $(k=1, q=2)$ as well as maximal gaps between prime $k$-tuples $(k \geq 2, q=2)$. One of our present goals is to generalize Formulas (1) and (2) to gaps between primes in a residue class-and test them in computational experiments. Another goal is to investigate how many maximal gaps should be expected between primes $p \leq x$ in a residue class, with an additional (optional) condition that $p$ starts a prime constellation of a certain type.

\subsection{Notation}

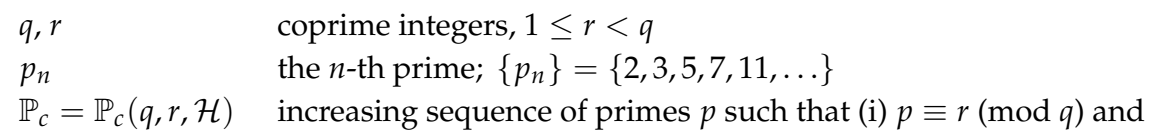




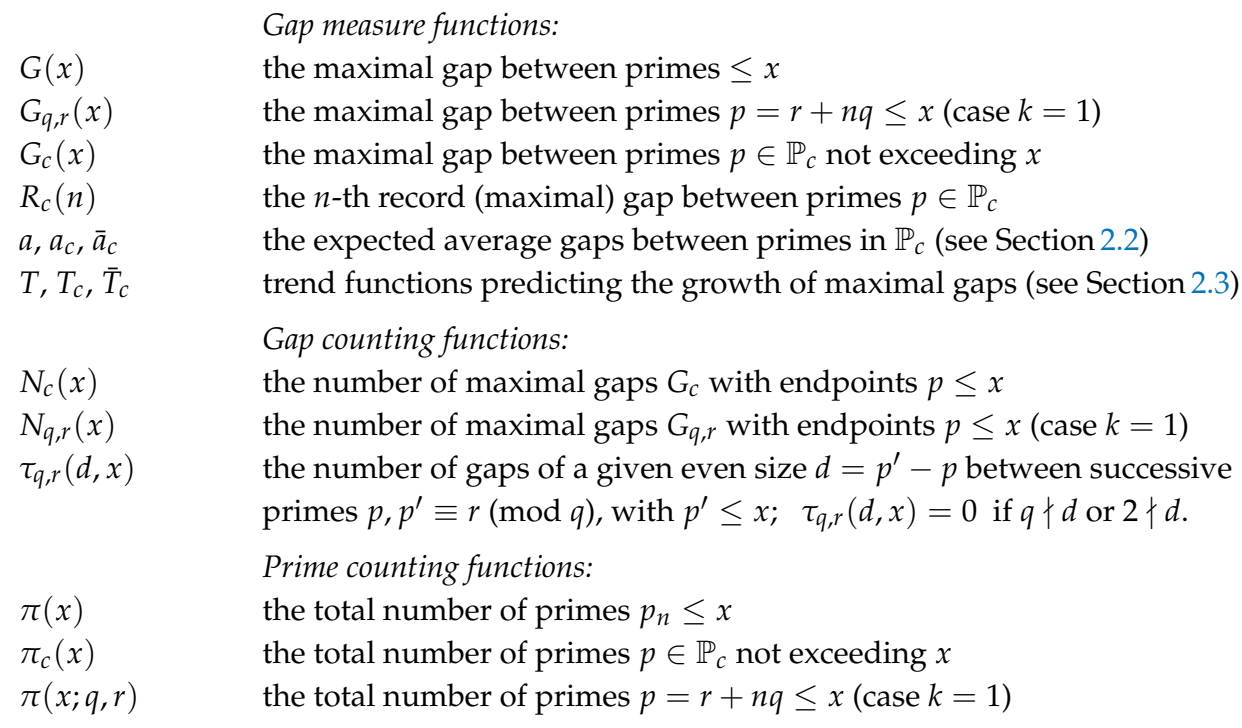

Quantities with the $c$ subscript may, in general, depend on $q, r, k$, and on the pattern of the prime $k$-tuple. However, average gaps $a, a_{c}, \bar{a}_{c}$ and trend functions $T, T_{c}, \bar{T}_{c}$ are independent of $r$. Expressions like $\pi_{c}^{2}(x)$ or $\log ^{2} x$ denote the square of the respective function.

\subsection{Definitions: Prime $k$-Tuples, Gaps, Sequence $\mathbb{P}_{c}$}

Prime $k$-tuples are clusters of $k$ consecutive primes that have an admissible pattern $\mathcal{H}$. A $k$-tuple is admissible (infinitely repeatable) unless it is prohibited by an elementary divisibility argument. For example, the cluster of five numbers $(p, p+2, p+4, p+6, p+8)$ is prohibited because one of the numbers is divisible by 5 (and, moreover, at least one of the numbers is divisible by 3 ); hence all these five numbers cannot simultaneously be prime infinitely often. Likewise, the cluster of three numbers $(p, p+2, p+4)$ is prohibited because one of the numbers is divisible by 3 ; so these three numbers cannot simultaneously be prime infinitely often. In what follows, when we speak of a $k$-tuple, for certainty we will mean a densest admissible prime $k$-tuple, with a given $k \leq 7$. However, our observations can be extended to other admissible $k$-tuples, including those with larger $k$ and not necessarily densest ones. The densest $k$-tuples that exist for a given $k$ may sometimes be called prime constellations or prime $k$-tuplets. Below are examples of prime $k$-tuples with $k=2,4,6$.

- Twin primes are pairs of consecutive primes that have the form $(p, p+2)$. This is the densest admissible pattern of two; $\mathcal{H}=(0,2)$.

- Prime quadruplets are clusters of four consecutive primes of the form $(p, p+2, p+6, p+8)$. This is the densest admissible pattern of four; $\mathcal{H}=(0,2,6,8)$.

- $\quad$ Prime sextuplets are clusters of six consecutive primes $(p, p+4, p+6, p+10, p+12, p+16)$. This is the densest admissible pattern of six; $\mathcal{H}=(0,4,6,10,12,16)$.

A gap between prime $k$-tuples is the distance $p^{\prime}-p$ between the initial primes $p$ and $p^{\prime}$ in two consecutive $k$-tuples of the same type (i.e., with the same pattern). For example, the gap between twin prime pairs $(17,19)$ and $(29,31)$ is $12: p^{\prime}-p=29-17=12$.

A maximal gap between prime $k$-tuples is a gap that is strictly greater than all gaps between preceding $k$-tuples of the same type. For example, the gap of size 6 between twin primes $(5,7)$ and $(11,13)$ is maximal, while the gap (also of size 6$)$ between twin primes $(11,13)$ and $(17,19)$ is not maximal. 
Let $q>r \geq 1$ be coprime integers. Let $\mathbb{P}_{c}=\mathbb{P}_{c}(q, r, \mathcal{H})$ be an increasing sequence of primes $p$ satisfying two conditions: (i) $p \equiv r(\bmod q)$ and (ii) $p$ starts a prime $k$-tuple with a given pattern $\mathcal{H}$. Importantly, $\mathbb{P}_{c}$ depends on $q, r, k$, and on the pattern of the $k$-tuple. When $k=1, \mathbb{P}_{c}$ is the sequence of all primes $p \equiv r(\bmod q)$. Gaps between primes in $\mathbb{P}_{c}$ are defined as differences $p^{\prime}-p$ between successive primes $p, p^{\prime} \in \mathbb{P}_{c}$. As before, a gap is maximal if it is strictly greater than all preceding gaps. Accordingly, for successive primes $p, p^{\prime} \in \mathbb{P}_{c}$ we define

$$
G_{c}(x)=\max _{\substack{p^{\prime}<x \\ p, p^{\prime} \in \mathbb{P}_{c}}}\left(p^{\prime}-p\right) .
$$

Studying maximal gaps between primes in $\mathbb{P}_{c}$ is convenient. Indeed, if the modulus $q$ used for defining $\mathbb{P}_{c}$ is "not too small", we get plenty of data to study maximal gaps; that is, we get many sequences of maximal gaps corresponding to $\mathbb{P}_{c}$ 's with different $r$ for the same $q$, which allows us to study common properties of these sequences. (One such property is the average number of maximal gaps between primes in $\mathbb{P}_{c}$ below $x$.) By contrast, data on maximal prime gaps are scarce: at present we know that there are only 80 maximal gaps between primes below $2^{64}$ [3]. Even fewer maximal gaps are known between $k$-tuples of any given type [17].

\section{Remark 1.}

(i) In Section 2 we derive formulas predicting the most probable sizes of maximal gaps $G_{c}(x)$. It is not known how close these most probable sizes might be to the maximal order of $G_{c}(x)$. Thus, in the special case $k=1, q=2, r=1$, probable values of $G(x)$ seem to be about $\log ^{2} x-2 \log x \log \log x$ [13]; but it is not implausible that the maximal order of $G(x)$ is closer to $2 e^{-\gamma} \log ^{2} x$ [6]. For further discussion of extremely large gaps, see Section 3.5 .

(ii) How hard is it to compute gaps in sequence $\mathbb{P}_{c}$ ? Given $k=1, q \approx 10^{3}$ and $r$ coprime to $q$, our PARI/GP code (Appendix A) takes several hours to compute all maximal gaps in sequence $\mathbb{P}_{c}$ up to 14-digit primes. In some numerical experiments, we carried out the computation all the way to $10^{14}$. In most cases, however, we stopped the computation at $e^{28}$ or at $10^{12}$ or even earlier, to quickly gather statistics for all $r$ coprime to q. A similar strategy was also used for sequences $\mathbb{P}_{c}$ with $k \geq 2$ (source code for $k \geq 2$ is not included). See Section 3 for a detailed discussion of our numerical results.

\subsection{Generalization to Other Subsets of Primes}

Sequences $\mathbb{P}_{c}$ include, as special cases, many different subsets of prime numbers: primes in a given residue class, twin primes, triplets, quadruplets, etc. However, formulas akin to (1) and (2) definitely have an even wider area of applicability. Namely, we expect that certain analogs of (1) or (2), possessing the general form

$$
\text { maximal gap size } \sim(\text { average gap near } x) \cdot L(x), \quad \text { with } L(x) \lesssim c \log x,
$$

will also be applicable to maximal gaps in the following subsets of primes:

- $\quad$ the sequence of prime-indexed primes [20], A006450

- $\quad$ higher iterates of prime-indexed primes [21-23], A038580

- $\quad$ primes $p=n^{2}+1, n \in \mathbb{N}[24], \underline{\mathrm{A} 002496}$

- $\quad$ primes $p=f(n)$, where $f(n)$ is an irreducible polynomial in $n$,

- primes in sequences of Beatty type: $p=\lfloor\beta n+\delta\rfloor, n \in \mathbb{N}$, for a fixed irrational $\beta>1$ and a fixed real $\delta[25], \underline{\mathrm{A} 132222}$.

The above list is by no means exhaustive, but it may serve as a starting point for future work. 


\subsection{When Are Equations (1), (2) Inapplicable?}

Analogs of Equations (1) and (2) are not applicable to sequences where (almost) every gap is maximal. Examples of this kind include:

- Mills primes [26], A051254,

- $\quad$ base- $B$ repunit primes [27], A076481,

- $\quad$ primes nearest to $e^{n}(\underline{\mathrm{A} 037028})$,

- in general, any sequence whose terms grow exponentially or super-exponentially.

\section{Heuristics and Conjectures}

We now focus on deriving analogs of Formulas (1) and (2) for sequences $\mathbb{P}_{c}=\mathbb{P}_{c}(q, r, \mathcal{H})$.

\subsection{Equidistribution of $k$-Tuples}

Everywhere we assume that $q>r$ are coprime positive integers. Let $\pi(x ; q, r)$ be the number of primes $p \equiv r(\bmod q)$ such that $p \leq x$. The prime number theorem for arithmetic progressions $[28,29]$ establishes that

$$
\pi(x ; q, r) \sim \frac{\operatorname{li} x}{\varphi(q)} \quad \text { as } x \rightarrow \infty .
$$

Furthermore, the generalized Riemann hypothesis (GRH) implies that

$$
\pi(x ; q, r)=\frac{\operatorname{li} x}{\varphi(q)}+O_{\varepsilon}\left(x^{1 / 2+\varepsilon}\right) \quad \text { for any } \varepsilon>0 .
$$

That is to say, the primes below $x$ are approximately equally distributed among the $\varphi(q)$ "allowed" residue classes (these classes form the reduced residue system modulo $q$ ). Roughly speaking, the GRH implies that, as $x \rightarrow \infty$, the numbers $\pi(x ; q, r)$ and $\lfloor\operatorname{li} x / \varphi(q)\rfloor$ almost agree in the left half of their digits.

Based on empirical evidence, below we conjecture that a similar phenomenon also occurs for prime $k$-tuples: in every $\mathcal{H}$-allowed residue class (as defined below in Section 2.1.1) there are infinitely many primes starting an admissible $k$-tuple with a particular pattern $\mathcal{H}$. Moreover, such primes are distributed approximately equally among all $\mathcal{H}$-allowed residue classes modulo $q$. Our conjectures are closely related to the Hardy-Littlewood $k$-tuple conjecture [30] and the Bateman-Horn conjecture [31].

\subsubsection{Counting the $\mathcal{H}$-Allowed Residue Classes}

Consider an example: take $\mathcal{H}=(0,2)$. Which residue classes modulo 4 may contain the lesser prime $p$ in a pair of twin primes $(p, p+2)$ ? Clearly, the residue class $0 \bmod 4$ is prohibited: all numbers in this class are even. The residue class $2 \bmod 4$ is prohibited for the same reason. The remaining residue classes, $p \equiv 1 \bmod 4$ and $p \equiv 3 \bmod 4$, are not prohibited. We call these two classes $\mathcal{H}$-allowed. Indeed, each of these two residue classes does contain lesser twin primes-and there are, conjecturally, infinitely many such primes in each class (see OEIS $\underline{\mathrm{A} 071695}$ and $\underline{\mathrm{A} 071698}$ ).

In general, given an admissible $k$-tuple with pattern $\mathcal{H}=\left(\Delta_{1}, \Delta_{2}, \ldots, \Delta_{k}\right)$, we say that a residue class $r(\bmod q)$ is $\mathcal{H}$-allowed if

$$
\operatorname{gcd}\left(r+\Delta_{1}, q\right)=\operatorname{gcd}\left(r+\Delta_{2}, q\right)=\operatorname{gcd}\left(r+\Delta_{3}, q\right)=\ldots=\operatorname{gcd}\left(r+\Delta_{k}, q\right)=1
$$

Thus a residue class is $\mathcal{H}$-allowed if it is not prohibited (by divisibility considerations) from containing infinitely many primes $p$ starting a prime $k$-tuple with pattern $\mathcal{H}$.

How many residue classes modulo $q$ are $\mathcal{H}$-allowed? To count them, we will need an appropriate generalization of Euler's totient $\varphi(q)$ : Golubev's totient functions [32-34]; see also [35], p. 289. 
Definition 1. Golubev's totient $\varphi_{k, \mathcal{H}}(q)$ is the number of $\mathcal{H}$-allowed residue classes modulo $q$ for a given pattern $\mathcal{H}=\left(\Delta_{1}, \ldots, \Delta_{k}\right)$. More formally,

$$
\varphi_{k, \mathcal{H}}(q)=\sum_{\substack{1 \leq x \leq q \\ \operatorname{gcd}\left(x+\Delta_{1}, q\right)=\cdots=\operatorname{gcd}\left(x+\Delta_{k}, q\right)=1}} 1 .
$$

Example 1. For prime quadruplets $(p, p+2, p+6, p+8)$ we have

$$
k=4, \quad \mathcal{H}=\left(\Delta_{1}, \Delta_{2}, \Delta_{3}, \Delta_{4}\right)=(0,2,6,8), \quad \text { and } \quad \varphi_{4, \mathcal{H}}(q)=\underline{A 319516}(q) .
$$

For instance, when $q=30$, we have $\varphi_{4, \mathcal{H}}(q)=1$ : indeed, there is only one residue class, namely, $p \equiv 11(\bmod 30)$ where divisibility considerations allow infinitely many primes $p$ at the beginning of prime quadruplets $(p, p+2, p+6, p+8)$.

Note that $\varphi_{1}(q)=\varphi(q)$ is Euler's totient function, A000010; and, for densest admissible $k$-tuples, $\varphi_{2, \mathcal{H}}(q)$ is A002472, see also Alder [36]; $\varphi_{3, \mathcal{H}}(q)$ is $\mathrm{A} 319534 ; \varphi_{4, \mathcal{H}}(q)$ is $\mathrm{A} 319516 ; \varphi_{5, \mathcal{H}}(q)$ is $\mathrm{A} 321029 ;$ and $\varphi_{6, \mathcal{H}}(q)$ is A321030. Like Euler's totient, the functions $\varphi_{k, \mathcal{H}}$ are multiplicative [33].

\subsubsection{The $k$-Tuple Infinitude Conjecture}

We expect each of the $\mathcal{H}$-allowed residue classes $r(\bmod q)$ to contain infinitely many primes $p$ starting admissible prime $k$-tuples with pattern $\mathcal{H}$. In other words, the corresponding sequence $\mathbb{P}_{c}=\mathbb{P}_{c}(q, r, \mathcal{H})$ is infinite.

\section{Remark 2.}

(i) The k-tuple infinitude conjecture generalizes Dirichlet's theorem on arithmetic progressions [37].

(ii) The conjecture follows from the Bateman-Horn conjecture [31].

\subsubsection{The $k$-Tuple Equidistribution Conjecture}

We conjecture that the number of primes $p \in \mathbb{P}_{c}, p \leq x$, is

$$
\pi_{c}(x)=\frac{C_{k, \mathcal{H}}}{\varphi_{k, \mathcal{H}}(q)} \mathrm{Li}_{k}(x)+O_{\eta, \mathcal{H}}\left(x^{\eta}\right) \quad \text { as } x \rightarrow \infty,
$$

where $\eta<1$, the coefficient $C_{k, \mathcal{H}}$ is the Hardy-Littlewood constant for the particular $k$-tuple (Appendix B), $\operatorname{Li}_{k}(x)=\int_{2}^{x} \log ^{-k} t d t$ (Appendix C), and $\varphi_{k, \mathcal{H}}(q)$ is Golubev's totient function (5).

\section{Remark 3.}

(i) Conjecture (6) is akin to the GRH-based Equation (4); the latter pertains to the case $k=1$.

(ii) The conjecture is compatible with the Bateman-Horn and Hardy-Littlewood k-tuple conjectures but does not follow from them.

(iii) It is plausible that, similar to (4), in (6) we can take $\eta=\frac{1}{2}+\varepsilon$ for any $\varepsilon>0$.

\subsection{Average Gap Sizes}

Consider a sequence $\mathbb{P}_{c}=\mathbb{P}_{c}(q, r, \mathcal{H})$, where the residue class $r(\bmod q)$ is $\mathcal{H}$-allowed. We define the expected average gaps between primes in $\mathbb{P}_{c}$ as follows.

Definition 2. The expected average gap between primes in $\mathbb{P}_{c}$ below $x$ is

$$
a_{\mathcal{c}}(x)=\frac{\varphi_{k, \mathcal{H}}(q)}{C_{k, \mathcal{H}}} \cdot \frac{x}{\mathrm{Li}_{k}(x)} .
$$


Definition 3. The expected average gap between primes in $\mathbb{P}_{c}$ near $x$ is

$$
\bar{a}_{c}(x)=\frac{\varphi_{k, \mathcal{H}}(q)}{C_{k, \mathcal{H}}} \cdot \log ^{k} x
$$

In view of the equidistribution conjecture (6), it is easy to see from these definitions that

$$
\frac{x}{\pi_{c}(x)} \approx a_{c}(x)<\bar{a}_{c}(x) \quad \text { for large } x
$$

We have the limits (with very slow convergence):

$$
\begin{gathered}
\lim _{x \rightarrow \infty} \frac{a_{c}(x)}{\bar{a}_{c}(x)}=1, \\
\lim _{x \rightarrow \infty} \frac{\bar{a}_{c}(x)-a_{c}(x)}{\bar{a}_{c}(x)} \cdot \log x=k .
\end{gathered}
$$

\subsection{Maximal Gap Sizes}

Recall that Formula (1) is applicable to the special case $q=2, k=1$ [12-14], while (2) is applicable to the special cases $q=2, k \geq 2$ [16]. We are now ready to generalize (1) and (2) for predicting maximal gaps between primes in sequences $\mathbb{P}_{c}$ with $q \geq 2$.

\subsubsection{Case of $k$-Tuples: $k \geq 2$}

Consider a probabilistic example. Suppose that intervals between rare random events are exponentially distributed, with $\operatorname{cdf} \operatorname{Exp}(\xi ; \alpha)=1-e^{-\xi / \alpha}$, where $\alpha$ is the mean interval between events. If our observations of the events continue for $x$ seconds, extreme value theory (EVT) predicts that the expected maximal interval between events is

$$
\text { expected maximal interval }=\alpha \log \frac{x}{\alpha}+O(\alpha)=\frac{x}{\Pi(x)} \log \Pi(x)+O(\alpha),
$$

where $\Pi(x) \approx x / \alpha$ is the total count of the events we observed in $x$ seconds. (For details on deriving Equation (11), see e.g., [38], pp. 114-116 or [16], Section 8.)

By analogy with EVT, we define the expected trend functions for maximal gaps as follows.

Definition 4. The lower trend of maximal gaps between primes in $\mathbb{P}_{c}$ is

$$
T_{\mathcal{c}}(x)=a_{c}(x) \cdot \log \frac{C_{k, \mathcal{H}} \operatorname{Li}_{k}(x)}{\varphi_{k, \mathcal{H}}(q)} .
$$

In view of the equidistribution conjecture (6),

$$
T_{c}(x) \approx a_{c}(x) \cdot \log \pi_{\mathcal{c}}(x) \approx \frac{x}{\pi_{c}(x)} \cdot \log \pi_{c}(x) \quad \text { as } x \rightarrow \infty .
$$

We also define another trend function, $\bar{T}_{\mathcal{c}}(x)$, which is simpler because it does not use $\operatorname{Li}_{k}(x)$.

Definition 5. The upper trend of maximal gaps between primes in $\mathbb{P}_{c}$ is

$$
\bar{T}_{c}(x)=\bar{a}_{c}(x) \cdot \log \frac{x}{\bar{a}_{c}(x)} .
$$


The above definitions imply that

$$
T_{\mathcal{c}}(x)<\bar{T}_{\mathcal{c}}(x)<C_{k, \mathcal{H}}^{-1} \varphi_{k, \mathcal{H}}(q) \cdot \log ^{k+1} x \quad \text { for large } x .
$$

At the same time, we have the asymptotic equivalence:

$$
T_{\mathcal{c}}(x) \sim \bar{T}_{\mathcal{c}}(x) \sim C_{k, \mathcal{H}}^{-1} \varphi_{k, \mathcal{H}}(q) \cdot \log ^{k+1} x \quad \text { as } x \rightarrow \infty .
$$

We have the limits (convergence is quite slow):

$$
\begin{gathered}
\lim _{x \rightarrow \infty} \frac{\bar{T}_{\mathcal{c}}(x)-T_{\mathcal{c}}(x)}{\bar{a}_{\mathcal{c}}(x)}=k, \\
\lim _{x \rightarrow \infty} \frac{C_{k, \mathcal{H}}^{-1} \varphi_{k, \mathcal{H}}(q) \log ^{k+1} p-\bar{T}_{\mathcal{C}}(x)}{\bar{a}_{\mathcal{c}}(x) \log \log x}=k .
\end{gathered}
$$

Therefore, $\bar{T}_{\mathcal{c}}(x)-T_{\mathcal{c}}(x)=O_{k}\left(\bar{a}_{c}\right)$, while $C_{k, \mathcal{H}}^{-1} \varphi_{k, \mathcal{H}}(q) \log ^{k+1} p-\bar{T}_{\mathcal{c}}(x)=O_{k}\left(\bar{a}_{c} \log \log x\right)$. We make the following conjectures regarding the behavior of maximal gaps $G_{c}(x)$.

Conjecture on the trend of $G_{c}(x)$. For any sequence $\mathbb{P}_{c}$ with $k \geq 2$, a positive proportion of maximal gaps $G_{\mathcal{C}}(x)$ satisfy the double inequality

$$
T_{\mathcal{c}}(x) \lesssim G_{c}(x) \lesssim \bar{T}_{\mathcal{c}}(x) \quad \text { as } x \rightarrow \infty,
$$

and the difference $G_{\mathcal{C}}(x)-\bar{T}_{\mathcal{c}}(x)$ changes its sign infinitely often.

Generalized Cramér conjecture for $G_{c}(p)$. Almost all maximal gaps $G_{c}(p)$ satisfy

$$
G_{c}(p)<C_{k, \mathcal{H}}^{-1} \varphi_{k, \mathcal{H}}(q) \log ^{k+1} p
$$

Generalized Shanks conjecture for $G_{c}(p)$. Almost all maximal gaps $G_{c}(p)$ satisfy

$$
G_{c}(p) \sim C_{k, \mathcal{H}}^{-1} \varphi_{k, \mathcal{H}}(q) \log ^{k+1} p \quad \text { as } p \rightarrow \infty .
$$

Here $G_{c}(p)$ denotes the maximal gap that ends at the prime $p$.

\subsubsection{Case of Primes: $k=1$}

The EVT-based trend Formulas (12) and (14) work well for maximal gaps between $k$-tuples, $k \geq 2$. However, when $k=1$, the observed sizes of maximal gaps $G_{q, r}(x)$ between primes in residue class $r$ $\bmod q$ are usually a little less than predicted by the corresponding lower trend formula akin to (12). For example, with $k=1$ and $q=2$, the most probable values of maximal prime gaps $G(x)$ turn out to be less than the EVT-predicted value $\frac{x \log \operatorname{li} x}{\operatorname{li} x}-$ less by approximately $\log x \log \log x$ (cf. Cadwell [15]), p. 912. In this respect, primes do not behave like "random darts". Instead, the situation looks as if primes "conspire together" so that each prime $p_{n} \leq x$ lowers the typical maximal gap $G(x)$ by about $p_{n}^{-1} \log x$; indeed, we have $\sum_{p_{n} \leq x} p_{n}^{-1} \sim \log \log x$. Below we offer a heuristic explanation of this phenomenon.

Let $\tau_{q, r}(d, x)$ be the number of gaps of a given even size $d=p^{\prime}-p$ between successive primes $p, p^{\prime} \equiv r(\bmod q), p^{\prime} \leq x$. Empirically, the function $\tau_{q, r}$ has the form (cf. $\left.[13,39,40]\right)$

$$
\tau_{q, r}(d, x) \approx P_{q}(d) B_{q}(x) e^{-d \cdot A_{q}(x)},
$$

where $P_{q}(d)$ is an oscillating factor (encoding a form of singular series), and

$$
\tau_{q, r}(d, x)=P_{q}(d)=0 \quad \text { if } q \nmid d \text { or } 2 \nmid d .
$$


The essential point now is that we can find the unknown functions $A_{q}(x)$ and $B_{q}(x)$ in (22) just by assuming the exponential decay of $\tau_{q, r}$ as a function of $d$ and employing the following two conditions (which are true by definition of $\tau_{q, r}$ ):

$$
\begin{aligned}
& \text { (a) the total number of gaps is } \sum_{d=2}^{G_{q, r}(x)} \tau_{q, r}(d, x) \approx \pi(x ; q, r) \text {; } \\
& \text { (b) the total length of gaps is } \sum_{d=2}^{G_{q, r}(x)} d \cdot \tau_{q, r}(d, x) \approx x .
\end{aligned}
$$

The erratic behavior of the oscillating factor $P_{q}(d)$ presents an obstacle in the calculation of sums (24) and (25). We will assume that, for sufficiently regular functions $f(d, x)$,

$$
\sum_{d} P_{q}(d) f(d, x) \approx s \sum_{d} f(d, x)
$$

where $s$ is such that, on average, $P_{q}(d) \approx s$; and the summation is for $d$ such that both sides of (26) are non-zero. Extending the summation in Equations (24), (25) to infinity, using (26), and writing

$$
d=c j, \quad j \in \mathbb{N}, \quad c=\operatorname{LCM}(2, q)=O(q), \quad s=\lim _{n \rightarrow \infty} \frac{1}{n} \sum_{j=1}^{n} P_{q}(c j),
$$

we obtain two series expressions: (24) gives us a geometric series

$$
\sum_{d=2}^{\infty} \tau_{q, r}(d, x) \approx s B_{q}(x) \sum_{j=1}^{\infty} e^{-c j A_{q}(x)}=s B_{q}(x) \cdot \frac{e^{-c A_{q}(x)}}{1-e^{-c A_{q}(x)}} \approx \pi(x ; q, r),
$$

while (25) yields a differentiated geometric series

$$
\sum_{d=2}^{\infty} d \cdot \tau_{q, r}(d, x) \approx \operatorname{cs}_{q}(x) \sum_{j=1}^{\infty} j e^{-c j A_{q}(x)}=\operatorname{cs} B_{q}(x) \cdot \frac{e^{-c A_{q}(x)}}{\left(1-e^{-c A_{q}(x)}\right)^{2}} \approx x .
$$

Thus we have obtained two equations:

$$
s B_{q}(x) \cdot \frac{e^{-c A_{q}(x)}}{1-e^{-c A_{q}(x)}} \approx \pi(x ; q, r), \quad \operatorname{csB}_{q}(x) \cdot \frac{e^{-c A_{q}(x)}}{\left(1-e^{-c A_{q}(x)}\right)^{2}} \approx x .
$$

To solve these equations, we use the approximations $e^{-c A_{q}(x)} \approx 1$ and $1-e^{-c A_{q}(x)} \approx c A_{q}(x)$ (which is justified because we expect $A_{q}(x) \rightarrow 0$ for large $x$ ). In this way we obtain

$$
A_{q}(x) \approx \frac{\pi(x ; q, r)}{x}, \quad B_{q}(x) \approx \frac{c \pi^{2}(x ; q, r)}{s x} .
$$

A posteriori we indeed see that $A_{q}(x) \rightarrow 0$ as $x \rightarrow \infty$. Substituting (29) into (22) we get

$$
\tau_{q, r}(d, x) \approx P_{q}(d) \frac{c \pi^{2}(x ; q, r)}{s x} e^{-d \cdot \pi(x ; q, r) / x}
$$

From (30) we can obtain an approximate formula for $G_{q, r}(x)$. Note that $\tau_{q, r}(d, x)=1$ when the gap of size $d$ is maximal-in which case we have $d=G_{q, r}(x)$. So, to get an approximate value of the maximal gap $G_{q, r}(x)$, we solve for $d$ the equation $\tau_{q, r}(d, x)=1$, or

$$
\frac{c \pi^{2}(x ; q, r)}{x} e^{-d \cdot \pi(x ; q, r) / x} \approx 1,
$$


where we skipped $P_{q}(d) / s$ because, on average, $P_{q}(d) \approx s$. Taking the log of both sides of (31) we find the solution $G_{q, r}(x)$ expressed directly in terms of $\pi(x ; q, r)$ :

$$
G_{q, r}(x) \approx \frac{x}{\pi(x ; q, r)} \cdot\left(\log \frac{\pi^{2}(x ; q, r)}{x}+\log c\right) .
$$

Since $\pi(x ; q, r) \approx \frac{\operatorname{li} x}{\varphi(q)}$ and $\log \frac{\pi^{2}(x ; q, r)}{x} \approx 2 \log \frac{\operatorname{li} x}{\varphi(q)}-\log x$, we can state the following

Conjecture on the trend of $G_{q, r}(x)$. The most probable sizes of maximal gaps $G_{q, r}(x)$ are near a trend curve $T(q, x)$ :

$$
G_{q, r}(x) \sim T(q, x)=\frac{\varphi(q) x}{\operatorname{li} x} \cdot\left(2 \log \frac{\operatorname{li} x}{\varphi(q)}-\log x+b\right),
$$

where $b=b(q, x)=O(\log q)$ tends to a constant as $x \rightarrow \infty$. The difference $G_{q, r}(x)-T(q, x)$ changes its sign infinitely often.

Further, we expect that the width of distribution of the maximal gaps near $x$ is $O_{q}(\log x)$; i.e., the width of distribution is on the order of the average gap $\varphi(q) \log x$ (see Section 3.2). On the other hand, for large $x$, the trend (33) differs from the line $\varphi(q) \log ^{2} x$ by $O_{q}(\log x \log \log x)$, that is, by much more than the average gap. This suggests natural generalizations of the Cramér and Shanks conjectures:

Generalized Cramér conjecture for $G_{q, r}(p)$. Almost all maximal gaps $G_{q, r}(p)$ satisfy

$$
G_{q, r}(p)<\varphi(q) \log ^{2} p
$$

Generalized Shanks conjecture for $G_{q, r}(p)$. Almost all maximal gaps $G_{q, r}(p)$ satisfy

$$
G_{q, r}(p) \sim \varphi(q) \log ^{2} p \quad \text { as } p \rightarrow \infty .
$$

Conjectures (34) and (35) can be viewed as particular cases of (20), (21) for $k=1$.

\subsection{How Many Maximal Gaps Are There?}

This section generalizes the heuristic reasoning of [41], Section 2.3. Let $R_{c}(n)$ be the size of the $n$-th record (maximal) gap between primes in $\mathbb{P}_{c}$. Denote by $N_{c}(x)$ the total number of maximal gaps observed between primes in $\mathbb{P}_{c}$ not exceeding $x$. Let $\ell=\ell(x ; q, \mathcal{H})$ be a continuous slowly varying function estimating mean $\left(N_{c}(e x)-N_{c}(x)\right)$, the average number of maximal gaps between primes in $\mathbb{P}_{c}$, with the upper endpoints $p^{\prime} \in[x, e x]$. For $x \rightarrow \infty$, we will heuristically argue that if the limit of $\ell$ exists, then the limit is $k+1$. Suppose that

$$
\lim _{x \rightarrow \infty} \frac{N_{c}(x)}{\log x}=\lim _{x \rightarrow \infty} \frac{\operatorname{mean}_{r} N_{c}(x)}{\log x}=\lim _{x \rightarrow \infty} \ell(x ; q, \mathcal{H})=\ell_{*}>0,
$$

and the limit $\ell_{*}$ is independent of $q$. Let $n$ be a "typical" number of maximal gaps up to $x$; our assumption $\lim _{x \rightarrow \infty} \ell=\ell_{*}$ means that

$$
n \sim \ell_{*} \log x \quad \text { as } x \rightarrow \infty .
$$

For large $n$, we can estimate the order of magnitude of the typical $n$-th maximal gap $R_{\mathcal{c}}(n)$ using the generalized Cramér and Shanks conjectures (20) and (21):

$$
R_{c}(n)=G_{c}(x) \lesssim C_{k, \mathcal{H}}^{-1} \varphi_{k, \mathcal{H}}(q) \log ^{k+1} x \sim C_{k, \mathcal{H}}^{-1} \varphi_{k, \mathcal{H}}(q) \frac{n^{k+1}}{\ell_{*}^{k+1}}
$$


Define $\Delta R_{c}(n)=R_{c}(n+1)-R_{c}(n)$. By Formula (37), for large $q$ and large $n$ we have

$$
\begin{aligned}
\operatorname{mean}_{r} & R_{c}(n) \sim C_{k, \mathcal{H}}^{-1} \varphi_{k, \mathcal{H}}(q) \frac{n^{k+1}}{\ell_{*}^{k+1}}, \\
\operatorname{mean}_{r} \Delta R_{c}(n) & =\operatorname{mean}_{r}\left(R_{c}(n+1)-R_{c}(n)\right) \\
& \sim \frac{C_{k, \mathcal{H}}^{-1} \varphi_{k, \mathcal{H}}(q)}{\ell_{*}^{k+1}} \cdot\left((n+1)^{k+1}-n^{k+1}\right) \\
& \sim \frac{C_{k, \mathcal{H}}^{-1} \varphi_{k, \mathcal{H}}(q)}{\ell_{*}^{k+1}} \cdot(k+1) n^{k},
\end{aligned}
$$

where the mean is taken over all $\mathcal{H}$-allowed residue classes; see Section 2.1.1. Combining this with (36), we find

$$
\underset{r}{\operatorname{mean}} \Delta R_{c}(n) \sim \frac{k+1}{\ell_{*}} \cdot C_{k, \mathcal{H}}^{-1} \varphi_{k, \mathcal{H}}(q) \log ^{k} x .
$$

On the other hand, heuristically we expect that, on average, two consecutive record gaps should differ by the "local" average gap (8) between primes in $\mathbb{P}_{c}$ :

$$
\operatorname{mean}_{r} \Delta R_{c}(n) \sim C_{k, \mathcal{H}}^{-1} \varphi_{k, \mathcal{H}}(q) \log ^{k} x \quad(\sim \text { average gap near } x)
$$

Together, Equations (38) and (39) imply that

$$
\ell_{*}=k+1 \text {. }
$$

Therefore, for large $x$ we should expect (see Sections 3.3 and 3.4; cf. [42])

$$
N_{c}(x) \sim(k+1) \log x \quad \text { as } x \rightarrow \infty
$$

Special cases. For the number $N_{q, r}$ of maximal gaps between primes $p \equiv r(\bmod q)$ we have

$$
N_{q, r}(x) \sim 2 \log x \quad \text { as } x \rightarrow \infty
$$

This is asymptotically equivalent to the following semi-empirical formula for the number of maximal prime gaps up to $x$ (i.e., for the special case $k=1, q=2$; see [43], Section 3.4; OEIS A005669):

$$
N_{2,1}(x) \sim 2 \log \operatorname{li} x \quad \text { as } x \rightarrow \infty .
$$

Formula (42) tells us that maximal prime gaps occur, on average, about twice as often as records in an i.i.d. random sequence of $\lfloor\operatorname{li} x\rfloor$ terms. Note also the following straightforward generalization of (42) giving a very rough estimate of $N_{q, r}(x)$ in the general case:

$$
N_{q, r}(x) \approx \max \left(0,2 \log \frac{\operatorname{li} x}{\varphi(q)}\right) .
$$

Computation shows that, for the special case of maximal prime gaps $G(x)$, Formula (42) works quite well. However, the more general Formula (43) usually overestimates $N_{q, r}(x)$. At the same time, the right-hand side of (43) is less than $2 \log x$. Thus the right-hand sides of (41) as well as (43) overestimate the actual gap counts $N_{q, r}(x)$ in most cases. 
In Section 3.3 we will see an alternative (a posteriori) approximation based on the average number of maximal gaps observed for primes in the interval $[x, e x]$. Namely, the estimated average number $\ell(x ; q, \mathcal{H})$ of maximal gaps with endpoints in $[x, e x]$ is

$$
\ell(x ; q, \mathcal{H}) \approx \operatorname{mean}_{r}\left(N_{c}(e x)-N_{c}(x)\right) \approx k+1-\frac{\kappa(q, \mathcal{H})}{\log x+\delta(q, \mathcal{H})} .
$$

\section{Numerical Results}

To test our conjectures of the previous section, we performed extensive computational experiments. We used PARI/GP (see Appendix A for code examples) to compute maximal gaps $G_{c}$ between initial primes $p=r+n q \in \mathbb{P}_{c}$ in densest admissible prime $k$-tuples, $k \leq 6$. We experimented with many different values of $q \in\left[4,10^{5}\right]$. To assemble a complete data set of maximal gaps for a given $q$, we used all $\mathcal{H}$-allowed residue classes $r(\bmod q)$. For additional details of our computational experiments with maximal gaps between primes $p=r+n q$ (i.e., for the case $k=1$ ), see also [43], Section 3. In this section we omit the subscript $\mathcal{H}$ in $\varphi_{k}$ and $C_{k}$ because we are working with densest $k$-tuples: for each $k=2,4,6$ there is only one densest pattern $\mathcal{H}$, while for each $k=3,5,7$ there are two densest patterns $\mathcal{H}$, with equal numerical values of functions $\varphi_{k}(q)$ and equal Hardy-Littlewood constants $C_{k}$.

\subsection{The Growth Trend of Maximal Gaps}

The vast majority of maximal gap sizes $G_{\mathcal{C}}(x)$ are indeed observed near the trend curves predicted in Section 2.3. Specifically, for maximal gaps $G_{c}$ between primes $p=r+n q \in \mathbb{P}_{c}$ in $k$-tuples $(k \geq 2)$, the gap sizes are mostly found in the neighborhood of the corresponding trend curves of Equations (12) and (14) derived from extreme value theory. However, for $k=1$, the trend Equation (33) gives a better prediction of maximal gaps $G_{q, r}$.

Figures 1-3 illustrate our numerical results for $k=1,2,6, q=313$. The horizontal axis in these figures is $\log ^{k+1} p$ for end-of-gap primes $p$. Note that all gaps shown in the figures satisfy the generalized Cramér conjecture, i.e., inequalities (20), (34); for rare exceptions, see Section 3.5. Results for other values of $q$ look similar to Figures 1-3.

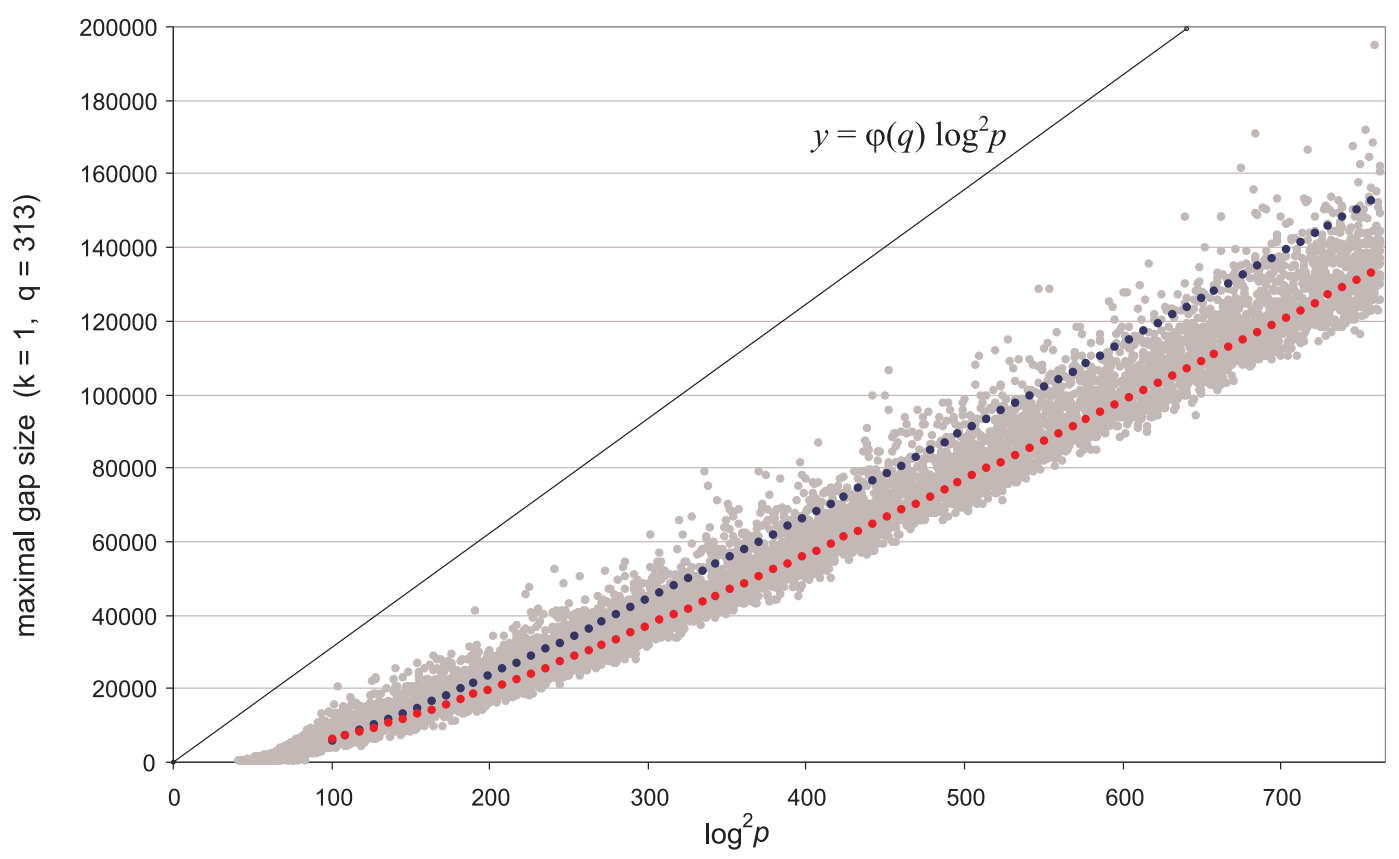

Figure 1. Maximal gaps $G_{q, r}$ between primes $p=r+n q \leq x$ for $q=313, x<10^{12}$. Red curve: trend (33), (45); blue curve: EVT-based trend $\frac{\varphi(q) x}{\operatorname{li} x} \log \frac{\operatorname{li} x}{\varphi(q)}$; top line: $y=\varphi(q) \log ^{2} p$. 


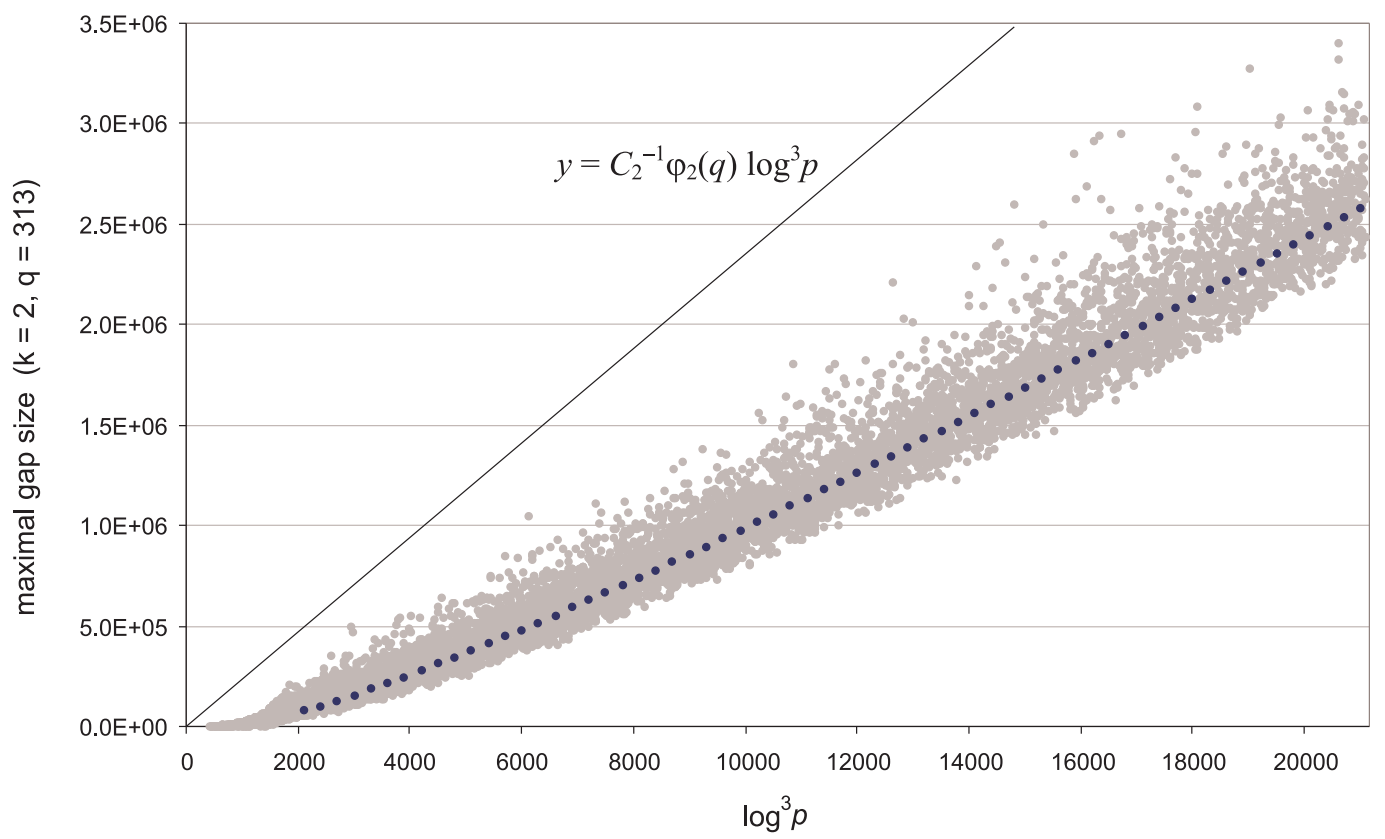

Figure 2. Maximal gaps $G_{c}$ between lesser twin primes $p=r+n q \in \mathbb{P}_{c}$ below $x$ for $q=313, x<10^{12}$, $k=2$. Dotted curve: trend $T_{c}$ of Equation (12); top line: $y=C_{2}^{-1} \varphi_{2}(q) \log ^{3} p$.

Numerical evidence suggests that

- For $k=1$ (the case of maximal gaps $G_{q, r}$ between primes $p=r+n q$ ) the EVT-based trend curve $\frac{\varphi(q) x}{\operatorname{li} x} \log \frac{\operatorname{li} x}{\varphi(q)}$ goes too high (Figure 1, blue curve). Meanwhile, the trend (33) (Figure 1, red curve)

$$
T(q, x)=\frac{\varphi(q) x}{\operatorname{li} x} \cdot\left(2 \log \frac{\operatorname{li} x}{\varphi(q)}-\log x+b\right)
$$

satisfactorily predicts gap sizes $G_{q, r}(x)$, with the empirical correction term

$$
b=b(q, x) \approx\left(b_{0}+\frac{b_{1}}{(\log \log x)^{b_{2}}}\right) \log \varphi(q) \asymp \log \varphi(q),
$$

where the parameter values

$$
b_{0}=1, \quad b_{1}=4, \quad b_{2}=2.7
$$

are close to optimal for $q \in\left[10^{2}, 10^{5}\right]$ and $x \in\left[10^{7}, 10^{14}\right]$. Here the qualifier optimal is to be understood in conjunction with the rescaling transformation (47) introduced below in Section 3.2. A trend $T(q, x)$ is optimal if after transformation (47) the most probable rescaled values $w$ turn out to be near zero, and the mode of best-fit Gumbel distribution for $w$-values is also close to zero, $\mu \approx 0$; see Figure 4 . In view of (45) it is possible that, for all $q$, the optimal term $b$ in (33) has the form $b(q, x)=(1+\beta(q, x)) \cdot \log \varphi(q) \sim \log \varphi(q)$, where $\beta(q, x)$ very slowly decreases to zero as $x \rightarrow \infty$. (Note that in Section 2.3.2 we correctly estimated $b$ to be $O(\log q)$ but did not predict the appearance of Euler's function $\varphi(q)$ in the term $b$.)

- For $k=2$, approximately half of maximal gaps $G_{c}$ between lesser twin primes $p \in \mathbb{P}_{c}$ are below the lower trend curve $T_{\mathcal{c}}(x)$ of Equation (12), while the other half are above that curve; see Figure 2.

- For $k \geq 3$, more than half of maximal gaps $G_{c}$ are usually above the lower trend curve $T_{c}(x)$ of Equation (12). At the same time, more than half of maximal gaps are usually below the upper 
trend curve $\bar{T}_{\mathcal{c}}(x)$ of Equation (14); see Figure 3. Recall that the two trend curves $T_{\mathcal{c}}$ and $\bar{T}_{\mathcal{c}}$ are within $k \bar{a}_{c}$ from each other as $x \rightarrow \infty$; see (17).

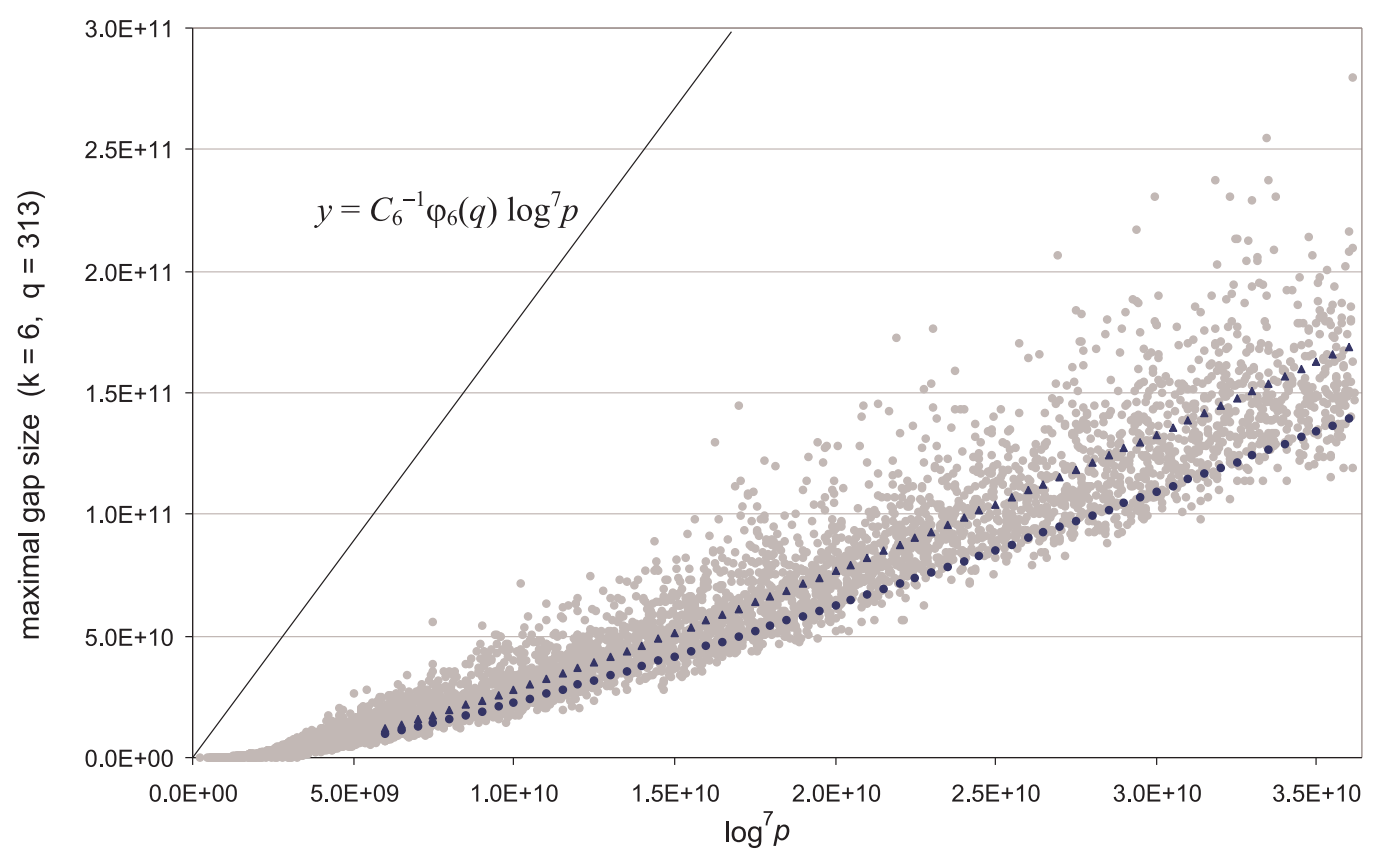

Figure 3. Maximal gaps $G_{c}$ between prime sextuplets $p=r+n q \in \mathbb{P}_{c}$ below $x$ for $q=313, x<10^{14}$, $k=6$. Dotted curves: trends $T_{\mathcal{c}}(\bullet)$ and $\bar{T}_{\mathcal{c}}(\mathbf{\Lambda})$ of Equations (12) and (14); top line: $y=C_{6}^{-1} \varphi_{6}(q) \log ^{7} p$.

As noted by Brent [44], twin primes seem to be more random than primes. We can add that, likewise, maximal gaps $G_{q, r}$ between primes in a residue class seem to be somewhat less random than those for prime $k$-tuples; primes $p \equiv r(\bmod q)$ do not go quite as far from each other as we would expect based on extreme value theory. Pintz [45] discusses various other aspects of the "random" and not-so-random behavior of primes.

\subsection{The Distribution of Maximal Gaps}

In Section 3.1 we have tested equations that determine the growth trend of maximal gaps between primes in sequences $\mathbb{P}_{c}$. How are maximal gap sizes distributed in the neighborhood of their respective trend?

We will perform a rescaling transformation (motivated by extreme value theory): subtract the trend from the actual gap size, and then divide the result by a natural unit, the "local" average gap. This way each maximal gap size is mapped to its rescaled value:

$$
\text { maximal gap size } G \mapsto \text { rescaled value }=\frac{G-\text { trend }}{\text { average gap }}
$$

Gaps above the trend curve are mapped to positive rescaled values, while gaps below the trend curve are mapped to negative rescaled values.

Case $k=1$. For maximal gaps $G_{q, r}$ between primes $p \equiv r(\bmod q)$, the trend function $T$ is given by Equations (33), (45) and (46). The rescaling operation has the form

$$
G_{q, r}(x) \mapsto w=\frac{G_{q, r}(x)-T(q, x)}{a(q, x)} .
$$

where $a(q, x)=\frac{\varphi(q) x}{\operatorname{li} x}$. Figure 4 shows histograms of rescaled values $w$ for maximal gaps $G_{q, r}$ between primes $p \equiv r(\bmod q)$ for $q=16001$. 
Case $k \geq 2$. For maximal gaps $G_{c}$ between prime $k$-tuples with $p=r+n q \in \mathbb{P}_{c}$, we can use the trend $T_{C}$ of Equation (12). Then the rescaling operation has the form

$$
G_{c}(x) \mapsto h=\frac{G_{c}(x)-T_{c}(x)}{a_{c}(x)},
$$

where $a_{c}(x)$ is defined by (7). Figure 5 shows histograms of rescaled values $h$ for maximal gaps $G_{\mathcal{C}}$ between lesser twin primes $p=r+n q \in \mathbb{P}_{c}$ for $q=16001, k=2$.
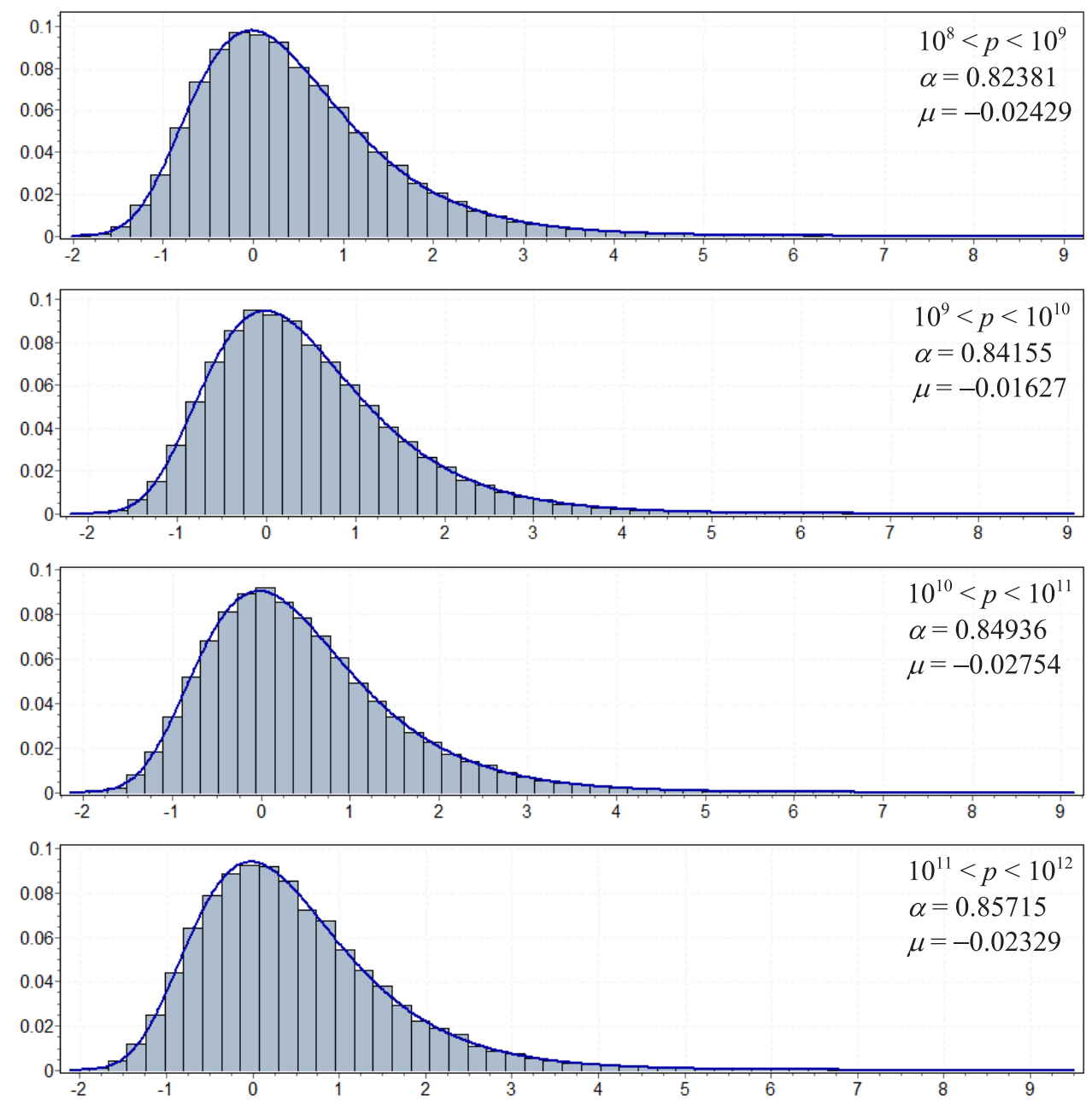

Figure 4. Histograms of $w$-values (47) for maximal gaps $G_{q, r}$ between primes $p=r+n q$ for $q=16001$, $r \in[1,16000]$. Curves are best-fit Gumbel distributions (pdfs) with scale $\alpha$ and mode $\mu$.

In both Figures 4 and 5, note that the histograms and fitting distributions are skewed to the right, i.e., the right tail is longer and heavier. Among two-parameter distributions, the Gumbel extreme value distribution is a very good fit; cf. [46,47]. This was true in all our computational experiments. 

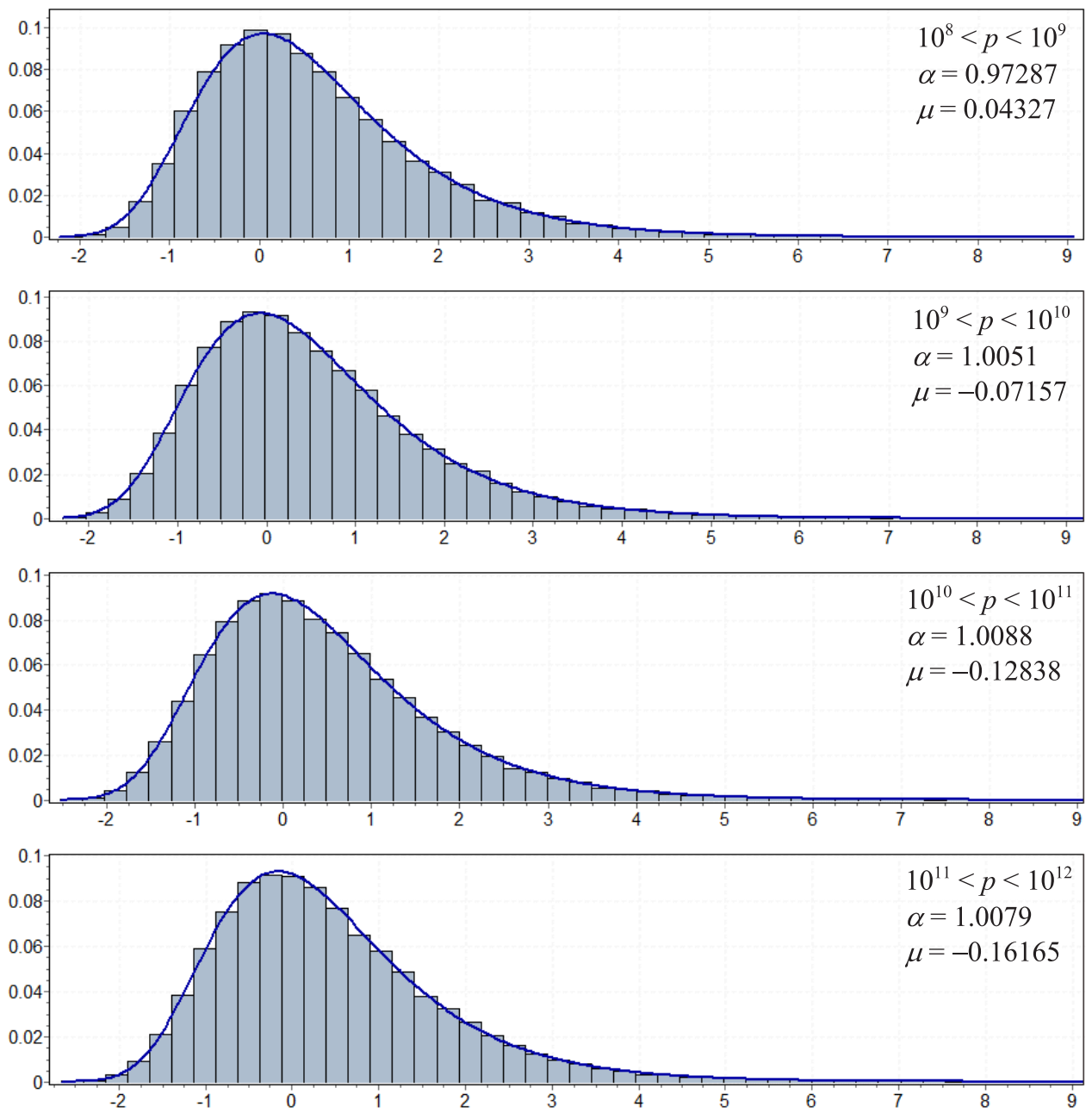

Figure 5. Histograms of $h$-values (48) for maximal gaps $G_{c}$ between lesser twin primes $p=r+n q \in \mathbb{P}_{c}$ for $q=16001$ and $\mathcal{H}$-allowed residue classes $r \in[1,16000], r \neq 15999$. Curves are best-fit Gumbel distributions (pdfs) with scale $\alpha$ and mode $\mu$.

Remark 4. For all histograms shown in Figures 4 and 5, the Kolmogorov-Smirnov goodness-of-fit statistic is less than 0.01; in fact, for most of the histograms, the goodness-of-fit statistic is about 0.003.

If we look at three-parameter distributions, then an excellent fit is the Generalized Extreme Value (GEV) distribution, which includes the Gumbel distribution as a special case. The shape parameter in the best-fit GEV distributions is close to zero; note that the Gumbel distribution is a GEV distribution whose shape parameter is exactly zero. So could the Gumbel distribution be the limit law for appropriately rescaled sequences of maximal gaps $G_{q, r}(p)$ and $G_{c}(p)$ as $p \rightarrow \infty$ ? Does such a limiting distribution exist at all?

The scale parameter $\alpha$. For $k=1$, we observed that the scale parameter of best-fit Gumbel distributions for $w$-values (47) was in the range $\alpha \in[0.7,1]$. The parameter $\alpha$ seems to slowly grow towards 1 as $p \rightarrow \infty$; see Figure 4. For $k \geq 2$, the scale parameter of best-fit Gumbel distributions for $h$-values (48) was usually a little over 1 ; see Figure 5 . However, if instead of (48) we use the (simpler) rescaling transformation

$$
G_{c}(x) \mapsto \bar{h}=\frac{G_{c}(x)-\bar{T}_{c}(x)}{\bar{a}_{c}(x)},
$$


where $\bar{a}_{\mathcal{C}}$ and $\bar{T}_{\mathcal{c}}$ are defined, respectively, by (8) and (14), then the resulting Gumbel distributions of $\bar{h}$-values will typically have scales $\alpha$ a little below 1 . In a similar experiment with random gaps, the scale was also close to 1; see [43], Section 3.3.

\subsection{Counting the Maximal Gaps}

We used PARI/GP function findallgaps (see source code in Appendix A.2) to determine average numbers of maximal gaps $G_{q, r}$ between primes $p=r+n q, p \in[x, e x]$, for $x=e^{j}, j=1,2, \ldots, 27$. Similar statistics were also gathered for gaps $G_{c}$. Figures 6-9 show the results of this computation for $q=16001, k \leq 4$. When $x$ is large, the average number of maximal gaps $G_{c}$ for $p \in[x, e x]$ indeed seems to very slowly approach $k+1$, as predicted by Equation (40). When $x$ is small $(x<q / e)$, there is at most one prime $p \in[2, e x]$ in sequence $\mathbb{P}_{c}$-and often there are no such primes at all; accordingly, we see no gaps ending in $[x, e x]$, and the corresponding plot points in Figures 6-9 are zero.

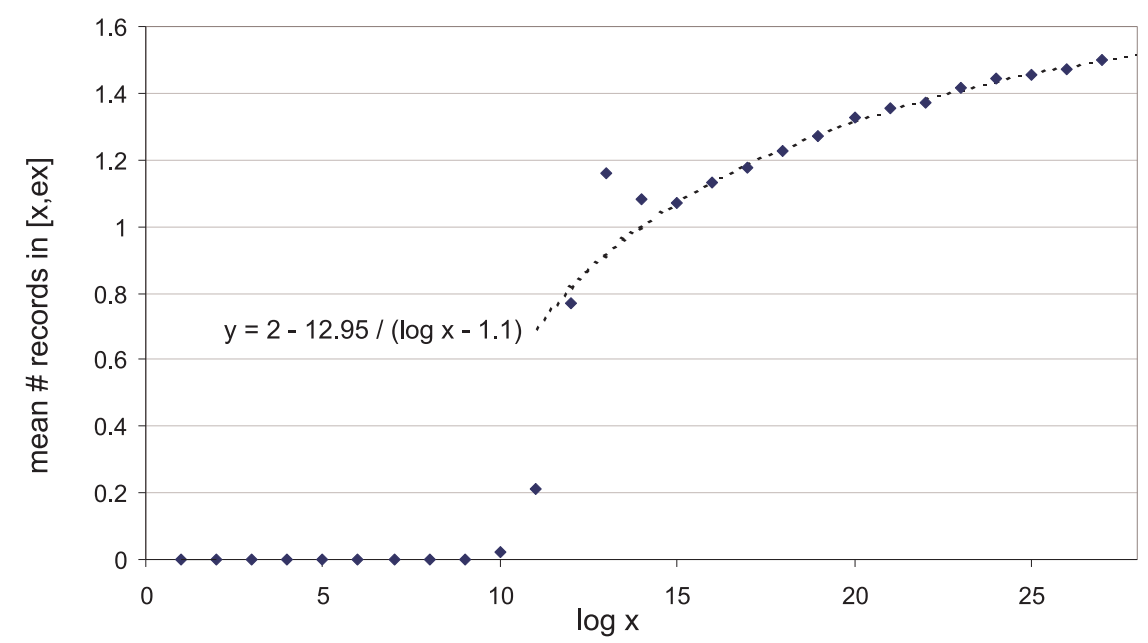

Figure 6. Primes $p=r+n q, k=1, q=16001$. Mean number of maximal gaps $G_{q, r}$ observed for $p \in[x, e x], x=e^{j}, j \leq 27$. Averaging for all $\mathcal{H}$-allowed $r$. Dotted curve is a hyperbola with horizontal asymptote $y=2$.

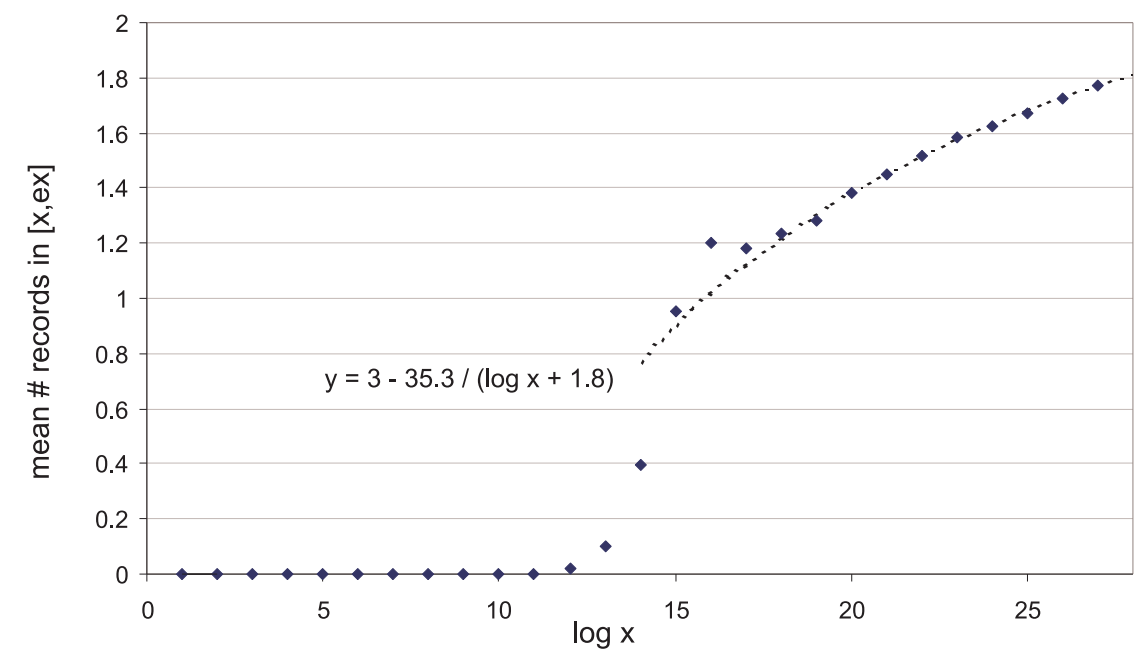

Figure 7. Lesser twin primes $p=r+n q \in \mathbb{P}_{c}, k=2, q=16001$. Mean number of maximal gaps $G_{c}$ observed for $p \in[x, e x], x=e^{j}, j \leq 27$. Averaging for all $\mathcal{H}$-allowed $r$. Dotted curve is a hyperbola with horizontal asymptote $y=3$. 


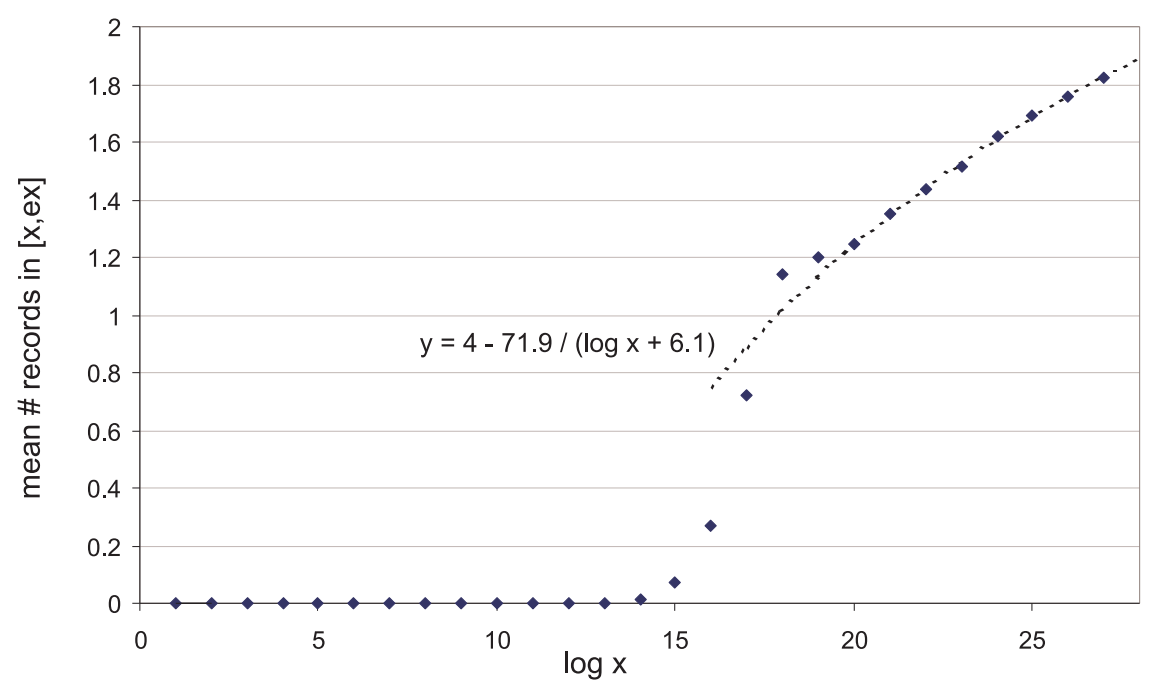

Figure 8. Prime triplets $(p, p+2, p+6), p=r+n q \in \mathbb{P}_{c}, k=3, q=16001$. Mean number of maximal gaps $G_{c}$ observed for $p \in[x, e x], x=e^{j}, j \leq 27$. Averaging for all $\mathcal{H}$-allowed $r$. Dotted curve is a hyperbola with horizontal asymptote $y=4$.

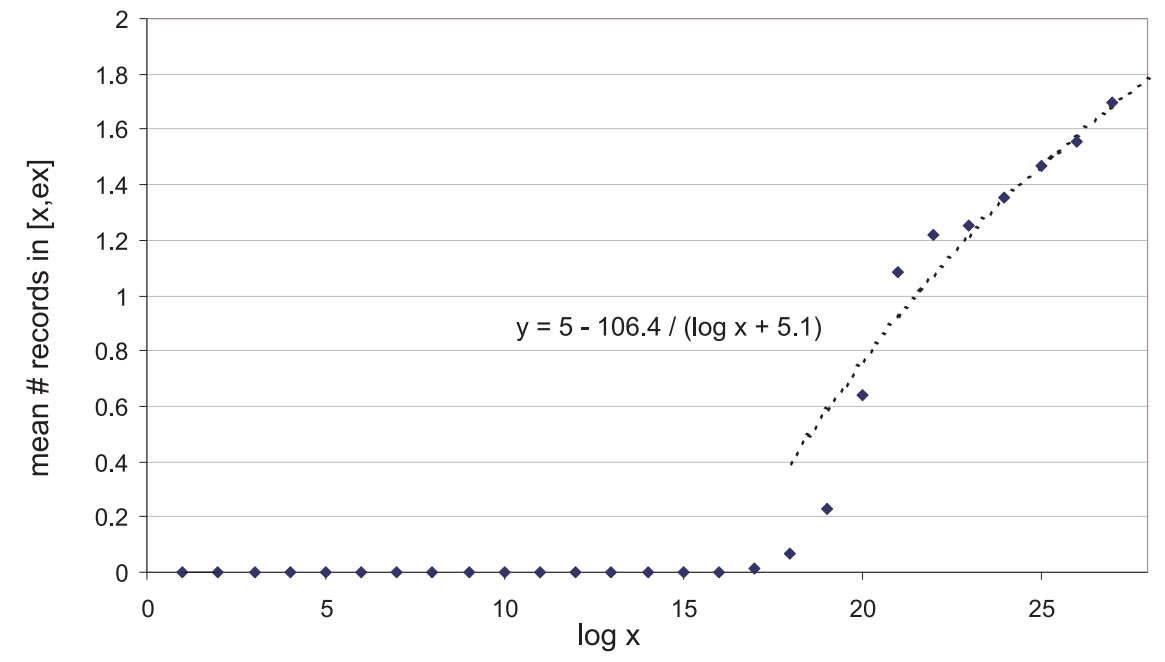

Figure 9. Prime quadruplets $(p, p+2, p+6, p+8), p=r+n q \in \mathbb{P}_{c}, k=4, q=16001$. Mean number of maximal gaps $G_{c}$ observed for $p \in[x, e x], x=e^{j}, j \leq 27$. Averaging for all $\mathcal{H}$-allowed $r$. Dotted curve is a hyperbola with horizontal asymptote $y=5$.

Starting from some $x_{0}>q / e$, the gap counts in $[x, e x]$ are no longer zero. Here we observe a "transition region" in which the mean number of maximal gaps $G_{c}(p)$ for primes $p \in[x, e x]$ grows from 0 to a little over 1 , while $x$ increases by about 3 orders of magnitude from $x_{0}$. The non-monotonic behavior of plot points in the transition region is explained, in part, by the fact that here the gap size may be comparable to the size of intervals $[x, e x]$. Then, for larger $x$, the typical number of gaps $G_{\mathcal{C}}$ between $k$-tuples continues to slowly increase; specifically, the graph of mean $\left(N_{\mathcal{c}}(e x)-N_{c}(x)\right)$ vs. $\log x$ is closely approximated by a hyperbola with horizontal asymptote $y=k+1$; see Equation (40) in Section 2.4. Why do the observed curves resemble hyperbolas? If we were working with random gaps, then perhaps the curves could be explained using the theory of records; cf. [42]. But primes are not random numbers; and so we simply have to treat the hyperbolas in Figures 6-9 as an experimental fact. 


\subsection{How Long Do We Wait for the Next Maximal Gap?}

Let $P(n)=\underline{\mathrm{A} 002386}(n)$ and $P^{\prime}(n)=\underline{\mathrm{A} 000101}(n)$ be the lower and upper endpoints of the $n$-th record (maximal) gap $R(n)$ between primes: $R(n)=\underline{\text { A005250 }}(n)=P^{\prime}(n)-P(n)$.

Consider the distances $P(n)-P(n-1)$ from one maximal gap to the next. (In statistics, a similar quantity is sometimes called "inter-record times"). In Figure 10 we present a plot of these distances; the figure also shows the corresponding plot for twin primes. As can be seen from Figure 10, the quantity $P(n)-P(n-1)$ grows approximately exponentially with $n$ (but not monotonically). Indeed, typical inter-record times are expected to satisfy

$$
\log (P(n)-P(n-1))<\log P(n) \sim \frac{n}{2} \quad \text { as } n \rightarrow \infty .
$$

The asymptotic equivalence $\sim$ in Equations (50) and (51) is a restatement of Equations (40) and (41). It would be logically unsound to suppose that $\log (P(n)-P(n-1)) \stackrel{?}{\sim} \log P(n)$ because we cannot exclude the possibility that $\log (P(n)-P(n-1))$ might (very rarely) become as small as $\log G(x) \approx 2 \log \log x$, where $x=P(n)$.

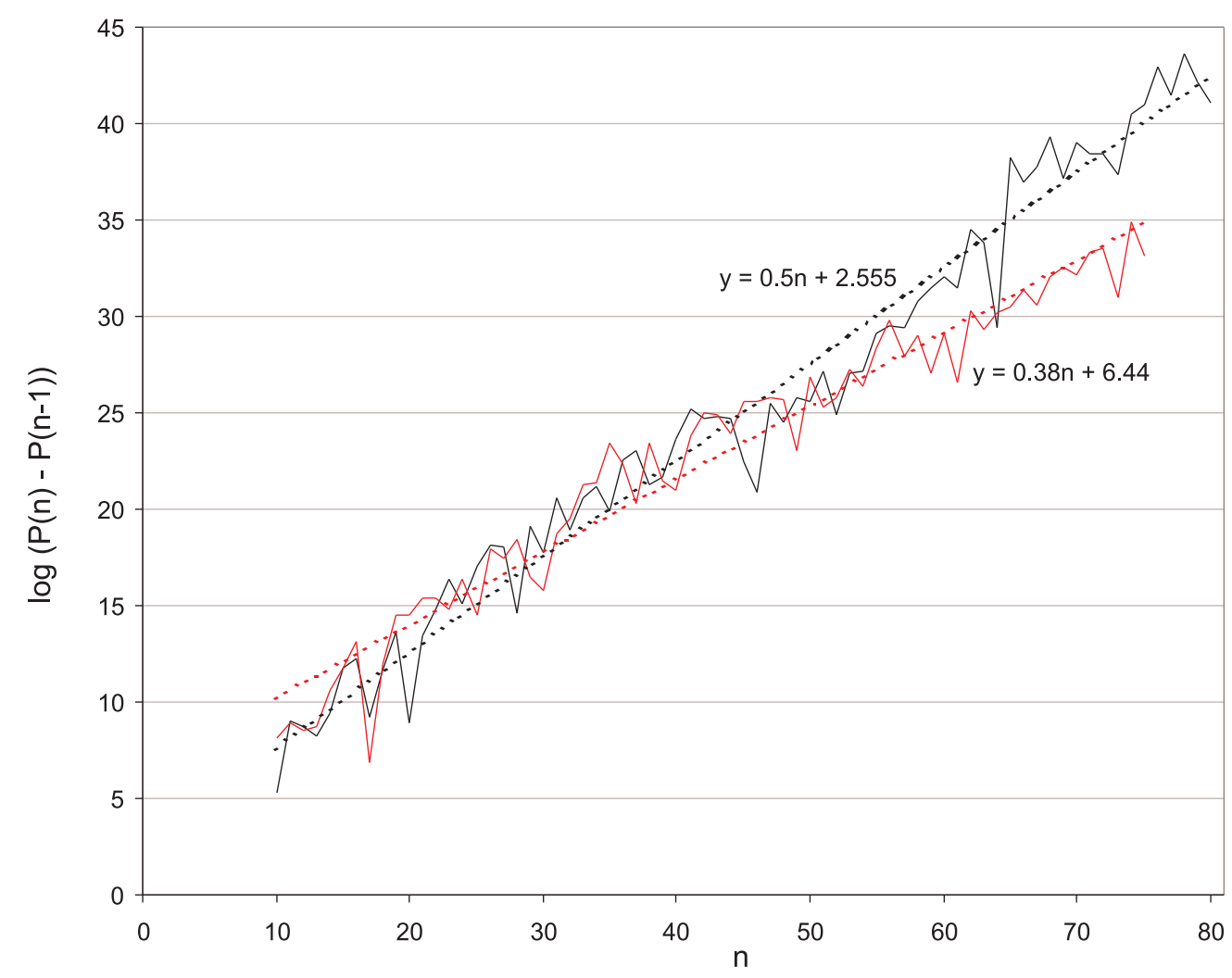

Figure 10. Inter-record times $P(n)-P(n-1)$ for gaps between primes (black) and a similar quantity $P_{c}(n)-P_{c}(n-1)$ for gaps between twin primes (red). Lines are exponential fits. Values for $n<10$ are skipped.

More generally, let $P_{c}(n)$ and $P_{c}^{\prime}(n)$ be the endpoints of the $n$-th maximal gap $R_{c}(n)$ between primes in sequence $\mathbb{P}_{c}$, where each prime is $r(\bmod q)$ and starts an admissible prime $k$-tuple. Then, in accordance with heuristic reasoning of Section 2.4, for typical inter-record times $P_{c}(n)-P_{c}(n-1)$ separating the maximal gaps $R_{c}(n-1)$ and $R_{c}(n)$ we expect to see

$$
\log \left(P_{\mathcal{c}}(n)-P_{\mathcal{c}}(n-1)\right)<\log P_{\mathcal{c}}(n) \sim \frac{n}{k+1} \quad \text { as } n \rightarrow \infty .
$$


In the special case $k=2$, that is, for maximal gaps between twin primes, the right-hand side of (51) is expected to be $\frac{n}{3}$ for large $n$ (whereas Figure 10 suggests the right-hand side $0.38 n$ based on a very limited data set for $10 \leq n \leq 75$ ). As we have seen in Section 3.3, the average number of maximal gaps between $k$-tuples occurring for primes $p \in[x, e x]$ slowly approaches $k+1$ from below. For moderate values of $x$ attainable in computation, this average is typically between 1 and $k+1$. Accordingly, we see that the right-hand side of (51) yields a prediction $\asymp e^{n /(k+1)}$ that underestimates the typical inter-record times and the primes $P_{c}(n)$. Computations may yield estimates

$$
P_{c}(n)-P_{c}(n-1)<P_{c}(n) \approx C e^{\beta n},
$$

where

$$
\beta \in\left[\frac{1}{k+1}, 1\right]
$$

with the estimated value of $\beta$ depending on the range of available data.

Remark 5. Sample graphs of $\log P_{c}(n)$ vs. $n$ can be plotted online at the OEIS website: click graph and scroll to the logarithmic plot for sequences $\underline{A 002386}(k=1), \underline{A 113275}(k=2), \underline{A 201597}(k=3), \underline{A 201599}(k=3)$, $\underline{A 229907}(k=4), \underline{A 201063}(k=5), \underline{A 201074}(k=5), \underline{A 200504}(k=6)$. In all these graphs, when $n$ is large enough, $\log P_{c}(n)$ seems to grow approximately linearly with $n$. We conjecture that the slope of such a linear approximation slowly decreases, approaching the slope value $1 /(k+1)$ as $n \rightarrow \infty$.

\subsection{Exceptionally Large Gaps: $G_{q, r}(p)>\varphi(q) \log ^{2} p$}

Recall that for the maximal prime gaps $G(x)$ Shanks [5] conjectured the asymptotic equality $G(x) \sim$ $\log ^{2} x$, a strengthened form of Cramér's conjecture. This seems to suggest that (unusually large) maximal gaps $g$ may in fact occur as early as at $x \asymp e^{\sqrt{g}}$. On the other hand, Wolf [48] conjectured that typically a gap of size $d$ appears for the first time between primes near $\sqrt{d} \cdot e^{\sqrt{d}}$. Combining these observations, we may further observe that exceptionally large maximal gaps, that is,

$$
\text { largest gaps } g=G(x)>\log ^{2} x
$$

are also those which appear for the first time unusually early. Namely, they occur at $x$ roughly by a factor of $\sqrt{d}$ earlier than the typical first occurrence of a gap $d$ at $x \asymp \sqrt{d} \cdot e^{\sqrt{d}}$. Note that Granville [6], p. 24, suggests that gaps of unusually large size (52) occur infinitely often-and we will even see infinitely many of those exceeding $1.1229 \log ^{2} x$. In contrast, Sun [49], Conj. 2.3, made a conjecture implying that exceptions like (52) occur only finitely often, while Firoozbakht's conjecture implies that exceptions (52) never occur for primes $p \geq 11$; see [50]. Here we cautiously predict that exceptional gaps of size (52) are only a zero proportion of maximal gaps. This can be viewed as restatement of the generalized Cramér conjectures (20), (34) for the special case $k=1, q=2$.

Table 1 lists exceptionally large maximal gaps $G_{q, r}(p)$ between primes $p \equiv r(\bmod q)$ for which inequality (34) does not hold:

$$
\text { largest gaps } G_{q, r}(p)>\varphi(q) \log ^{2} p \text {. }
$$

Three sections of Table 1 correspond to (i) odd $q, r$; (ii) even $q$; (iii) even $r$. (Overlap between sections is due to the fact that $\varphi(q)=\varphi(2 q)$ for odd $q$.) No other maximal gaps with this property were found for $p<10^{9}, q \leq 30000$. No such large gaps exist for $p<10^{10}, q \leq 1000$.

Remark 6. It is interesting that, for every gap listed in Table 1, at least one of the numbers $q$ and $r$ is composite. Thus far we have never seen a gap violating (34) with both $q$ and $r$ prime. 
Table 1. Exceptionally large maximal gaps: $G_{q, r}(p)>\varphi(q) \log ^{2} p$ for $p<10^{9}, r<q \leq 30000$.

\begin{tabular}{|c|c|c|c|c|c|c|}
\hline \multicolumn{2}{|c|}{ Gap $G_{q, r}(p)$} & \multirow{2}{*}{$\begin{array}{r}\text { Start of Gap } \\
3415781\end{array}$} & \multirow{2}{*}{$\begin{array}{r}\text { End of Gap }(p) \\
3624431\end{array}$} & \multirow{2}{*}{$\frac{q}{1605}$} & \multirow{2}{*}{$\frac{r}{341}$} & \multirow{2}{*}{$\frac{G_{q, r}(p) /\left(\varphi(q) \log ^{2} p\right)}{1.0786589153}$} \\
\hline (i) & 208650 & & & & & \\
\hline & 316790 & 726611 & 1043401 & 2005 & 801 & 1.0309808771 \\
\hline & 229350 & 1409633 & 1638983 & 2085 & 173 & 1.0145547849 \\
\hline & 532602 & 355339 & 887941 & 4227 & 271 & 1.0081862161 \\
\hline & 984170 & 5357381 & 6341551 & 4279 & 73 & 1.0339720553 \\
\hline & 1263426 & 10176791 & 11440217 & 4897 & 825 & 1.0056800570 \\
\hline & 2306938 & 82541821 & 84848759 & 6907 & 3171 & 1.0022590147 \\
\hline & 3415794 & 376981823 & 380397617 & 8497 & 3921 & 1.0703375544 \\
\hline & 2266530 & 198565889 & 200832419 & 8785 & 7319 & 1.0335372951 \\
\hline & 7326222 & 222677837 & 230004059 & 20017 & 8729 & 1.0166221904 \\
\hline & 6336090 & 10862323 & 17198413 & 23467 & 20569 & 1.0064940453 \\
\hline & 7230930 & 130172279 & 137403209 & 24595 & 15539 & 1.0468373915 \\
\hline & 5910084 & 51763573 & 57673657 & 28971 & 21367 & 1.0199911211 \\
\hline \multirow[t]{24}{*}{ (ii) } & 411480 & 470669167 & 471080647 & 3048 & 55 & 1.0235488825 \\
\hline & 208650 & 3415781 & 3624431 & 3210 & 341 & 1.0786589153 \\
\hline & 316790 & 726611 & 1043401 & 4010 & 801 & 1.0309808771 \\
\hline & 229350 & 1409633 & 1638983 & 4170 & 173 & 1.0145547849 \\
\hline & 657504 & 896016139 & 896673643 & 4566 & 2563 & 1.0179389550 \\
\hline & 1530912 & 728869417 & 730400329 & 6896 & 3593 & 1.0684247390 \\
\hline & 532602 & 355339 & 887941 & 8454 & 271 & 1.0081862161 \\
\hline & 984170 & 5357381 & 6341551 & 8558 & 73 & 1.0339720553 \\
\hline & 1263426 & 10176791 & 11440217 & 9794 & 825 & 1.0056800570 \\
\hline & 2119706 & 665152001 & 667271707 & 10046 & 6341 & 1.0223668231 \\
\hline & 1885228 & 163504573 & 165389801 & 10532 & 5805 & 1.0000704209 \\
\hline & 1594416 & 145465687 & 147060103 & 13512 & 9007 & 1.0026889378 \\
\hline & 2306938 & 82541821 & 84848759 & 13814 & 3171 & 1.0022590147 \\
\hline & 3108778 & 524646211 & 527754989 & 15622 & 12585 & 1.0098218219 \\
\hline & 1896608 & 164663 & 2061271 & 16934 & 12257 & 1.0598397341 \\
\hline & 3415794 & 376981823 & 380397617 & 16994 & 3921 & 1.0703375544 \\
\hline & 2266530 & 198565889 & 200832419 & 17570 & 7319 & 1.0335372951 \\
\hline & 2937868 & 71725099 & 74662967 & 17698 & 12803 & 1.0103309882 \\
\hline & 2823288 & 37906669 & 40729957 & 18098 & 9457 & 1.0162761199 \\
\hline & 2453760 & 11626561 & 14080321 & 18176 & 12097 & 1.0107626289 \\
\hline & 3906628 & 190071823 & 193978451 & 18692 & 11567 & 1.1480589845 \\
\hline & 2157480 & 13074917 & 15232397 & 27660 & 19397 & 1.0716522452 \\
\hline & 5450496 & 366870073 & 372320569 & 28388 & 11949 & 1.0140771094 \\
\hline & 3422630 & 735473 & 4158103 & 29762 & 21185 & 1.0368176014 \\
\hline \multirow[t]{14}{*}{ (iii) } & 657504 & 896016139 & 896673643 & 2283 & 280 & 1.0179389550 \\
\hline & 2119706 & 665152001 & 667271707 & 5023 & 1318 & 1.0223668231 \\
\hline & 3108778 & 524646211 & 527754989 & 7811 & 4774 & 1.0098218219 \\
\hline & 1896608 & 164663 & 2061271 & 8467 & 3790 & 1.0598397341 \\
\hline & 2937868 & 71725099 & 74662967 & 8849 & 3954 & 1.0103309882 \\
\hline & 2823288 & 37906669 & 40729957 & 9049 & 408 & 1.0162761199 \\
\hline & 3422630 & 735473 & 4158103 & 14881 & 6304 & 1.0368176014 \\
\hline & 3758772 & 144803717 & 148562489 & 15927 & 11360 & 1.0000152764 \\
\hline & 3002682 & 8462609 & 11465291 & 16869 & 11240 & 1.0107025944 \\
\hline & 8083028 & 344107541 & 352190569 & 19619 & 9900 & 1.1134625422 \\
\hline & 4575906 & 20250677 & 24826583 & 22653 & 21548 & 1.0463153374 \\
\hline & 5609136 & 34016537 & 39625673 & 26967 & 11150 & 1.0412524005 \\
\hline & 7044864 & 302145839 & 309190703 & 27519 & 14738 & 1.0048671503 \\
\hline & 6580070 & 9659921 & 16239991 & 28609 & 18688 & 1.0046426332 \\
\hline
\end{tabular}




\section{Summary}

We have extensively studied record (maximal) gaps between prime $k$-tuples in residue classes $(\bmod q)$. Our computational experiments described in Section 3 took months of computer time. Numerical evidence allows us to arrive at the following conclusions, which are also supported by heuristic reasoning.

- For $k=1$, the observed growth trend of maximal gaps $G_{q, r}(x)$ is given by (33) and (45). In particular, for maximal prime gaps $(k=1, q=2)$ the trend equation reduces to

$$
G_{2,1}(x) \sim T(2, x)=\log ^{2} x-2 \log x \log \log x+O(\log x) .
$$

- For $k \geq 2$, a significant proportion of maximal gaps $G_{c}(x)$ are observed between the trend curves of Equations (12) and (14), which can be heuristically derived from extreme value theory.

- The Gumbel distribution, after proper rescaling, is a possible limit law for $G_{q, r}(p)$ as well as $G_{c}(p)$. The existence of such a limiting distribution is an open question.

- Almost all maximal gaps $G_{q, r}(p)$ between primes in residue classes mod $q$ seem to satisfy appropriate generalizations of the Cramér and Shanks conjectures (34) and (35):

$$
G_{q, r}(p) \lesssim \varphi(q) \log ^{2} p
$$

- Similar generalizations (20) and (21) of the Cramér and Shanks conjectures are apparently true for almost all maximal gaps $G_{c}(p)$ between primes in $\mathbb{P}_{c}$ :

$$
G_{c}(p) \lesssim C_{k, \mathcal{H}}^{-1} \varphi_{k, \mathcal{H}}(q) \log ^{k+1} p .
$$

- Exceptionally large gaps $G_{q, r}(p)>\varphi(q) \log ^{2} p$ are extremely rare (Table 1 ). We conjecture that only a zero proportion of maximal gaps are such exceptions. A similar observation holds for $G_{c}(p)$ violating (20).

- We conjecture that the total number $N_{q, r}(x)$ of maximal gaps $G_{q, r}$ observed up to $x$ is below $C \log x$ for some $C>2$.

- More generally, we conjecture: the number $N_{c}(x)$ of maximal gaps between primes in $\mathbb{P}_{c}$ up to $x$ satisfies the inequality $N_{c}(x)<C \log x$ for some $C>k+1$, where $k$ is the number of integers in the pattern $\mathcal{H}$ defining the sequence $\mathbb{P}_{c}$.

Author Contributions: Conceptualization and methodology, A.K. and M.W.; software and visualization, A.K.; investigation, data curation, validation, and formal analysis, A.K. and M.W.; writing-original draft preparation, A.K.; writing-review and editing, A.K. and M.W.

Funding: This research received no external funding.

Acknowledgments: We are grateful to the anonymous referees for useful suggestions. Thanks also to all contributors and editors of the websites OEIS.org and PrimePuzzles.net.

Conflicts of Interest: The authors declare no conflict of interest.

\section{Abbreviations}

The following abbreviations are used in this manuscript:

cdf cumulative distribution function

pdf probability density function

EVT extreme value theory

GEV Generalized Extreme Value distribution

GRH Generalized Riemann Hypothesis 


\section{Appendix A. Details of Computational Experiments}

Interested readers can reproduce and extend our results using the programs below.

Appendix A.1. PARI/GP Program maxgap.gp

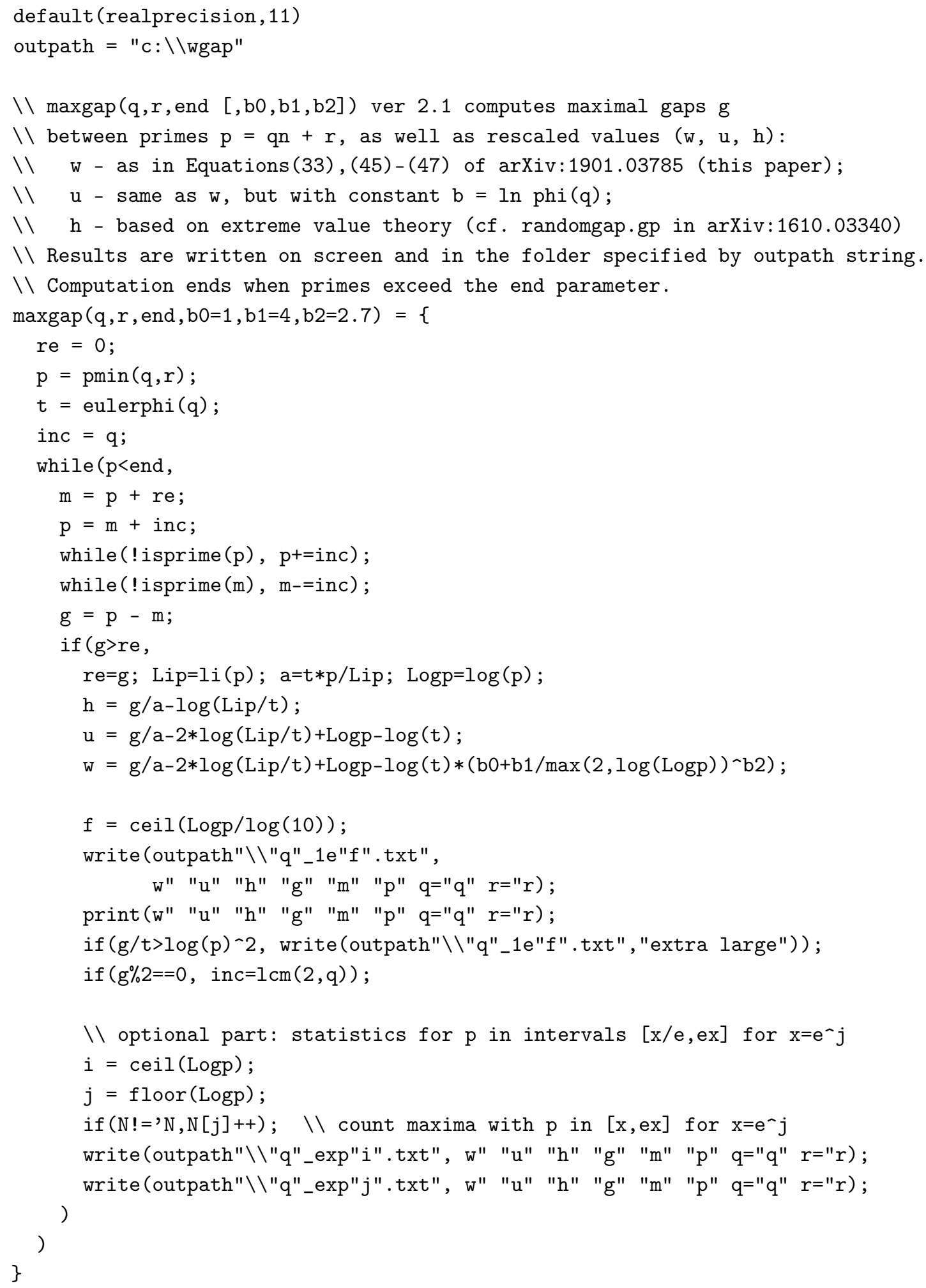


Appendix A.2. PARI/GP: Auxiliary Functions for maxgap.gp

1\These functions are intended for use with the program maxgap.gp

\I It is best to include them in the same file with maxgap.gp

$\backslash \backslash l i(x)$ computes the logarithmic integral of $x$

$\operatorname{li}(x)=\operatorname{real}(-\operatorname{eint} 1(-\log (x)))$

$\backslash \backslash \operatorname{pmin}(q, r)$ computes the least prime $p=q n+r$, for $n=0,1,2,3, \ldots$

$\operatorname{pmin}(q, r)=\operatorname{forstep}(p=r, 1 e 99, q$, if $(i \operatorname{sprime}(p), \operatorname{return}(p)))$

\\ findallgaps(q,end): Given $q$, call maxgap(q,r,end) for all $r$ coprime to $q$.

$\backslash \backslash$ Output total and average counts of maximal gaps in intervals [x,ex].

findallgaps $(q$, end $)=\{$

$\mathrm{t}=\operatorname{eulerphi}(\mathrm{q})$;

$\mathrm{N}=\operatorname{vector}(99, j, 0)$;

for $(r=1, q$, if $(\operatorname{gcd}(q, r)==1, \operatorname{maxgap}(q, r$, end $)))$;

$n \max =$ floor $(\log ($ end $))$;

for $(\mathrm{n}=1, \mathrm{nmax}$, $\operatorname{avg}=1.0 * \mathrm{~N}[\mathrm{n}] / \mathrm{t}$;

write(outpath"\\"q"stats.txt", n" "avg" "N[n]);

)

\}

Appendix A.3. Notes on Distribution Fitting

In order to study distributions of rescaled maximal gaps, we used the distribution-fitting software EasyFit [51]. Data files created with maxgap.gp are easily imported into EasyFit:

1. From the File menu, choose Open.

2. Select the data file.

3. Specify Field Delimiter $=$ space.

4. Click Update, then $O K$.

Caution: PARI/GP outputs large and small real numbers in a mantissa-exponent format with a space preceding the exponent (e.g., 1.7874829515 E-5), whereas EasyFit expects such numbers without a space (e.g., 1.7874829515E-5). Therefore, before importing into EasyFit, search the data files for " E" and replace all occurrences with "E".

\section{Appendix B. The Hardy-Littlewood Constants $C_{k, \mathcal{H}}$}

The Hardy-Littlewood $k$-tuple conjecture [30] allows one to predict the average frequencies of prime $k$-tuples near $p$, as well as the approximate total counts of prime $k$-tuples below $x$. Specifically, the Hardy-Littlewood $k$-tuple constants $C_{k, \mathcal{H}}$, divided by $\log ^{k} p$, give us an estimate of the average frequency of prime $k$-tuples near $p$ :

$$
\text { Frequency of } k \text {-tuples } \sim \frac{C_{k, \mathcal{H}}}{\log ^{k} p} .
$$

Accordingly ([52], pp. 61-68), for a given $k$-tuple pattern $\mathcal{H}$, the total count of $k$-tuples below $x$ is

$$
\pi_{k, \mathcal{H}}(x) \sim C_{k, \mathcal{H}} \int_{2}^{x} \frac{d t}{\log ^{k} t}=C_{k, \mathcal{H}} \operatorname{Li}_{k}(x) .
$$


The Hardy-Littlewood constants $C_{k, \mathcal{H}}$ can be defined in terms of infinite products over primes. In particular, for densest admissible prime $k$-tuples with $k \leq 7$ we have:

$$
\begin{aligned}
& C_{1}=1 \text { (by convention, in accordance with the prime number theorem); } \\
& C_{2, \mathcal{H}}=2 \prod_{p>2} \frac{p(p-2)}{(p-1)^{2}} \approx 1.32032363169373914785562422 \quad \text { (A005597, } \underline{\text { A114907); }} \\
& C_{3, \mathcal{H}}=\frac{9}{2} \prod_{p>3} \frac{p^{2}(p-3)}{(p-1)^{3}} \approx 2.85824859571922043243013466 \quad \text { (A065418); } \\
& C_{4, \mathcal{H}}=\frac{27}{2} \prod_{p>4} \frac{p^{3}(p-4)}{(p-1)^{4}} \approx 4.15118086323741575716528556 \quad \text { (A065419); } \\
& C_{5, \mathcal{H}}=\frac{15^{4}}{2^{11}} \prod_{p>5} \frac{p^{4}(p-5)}{(p-1)^{5}} \approx 10.131794949996079843988427 \quad \text { (A269843); } \\
& C_{6, \mathcal{H}}=\frac{15^{5}}{2^{13}} \prod_{p>6} \frac{p^{5}(p-6)}{(p-1)^{6}} \approx 17.2986123115848886061221077 \quad \text { (269846); } \\
& C_{7, \mathcal{H}}=\frac{35^{6}}{3 \cdot 2^{22}} \prod_{p>7} \frac{p^{6}(p-7)}{(p-1)^{7}} \approx 53.9719483001296523960730291 \quad \text { (A271742). }
\end{aligned}
$$

Forbes [53] gives values of the Hardy-Littlewood constants up to $k=24$, albeit with fewer significant digits; see also [54], p. 86. Starting from $k=8$, we may often encounter more than one numerical value of $C_{k, \mathcal{H}}$ for a single $k$. (If there are $m$ different patterns $\mathcal{H}$ of densest admissible prime $k$-tuples for the same $k$, then we typically have $\left\lceil\frac{m}{2}\right\rceil$ different numerical values of $C_{k, \mathcal{H}}$, depending on the actual pattern $\mathcal{H}$ of the $k$-tuple; see [53].)

\section{Appendix C. Integrals $\mathrm{Li}_{k}(x)$}

Let $k \in \mathbb{N}$ and $x>1$, and let

$$
\begin{aligned}
& F_{k}(x)=\int \frac{d x}{\log ^{k} x} \quad \text { (indefinite integral); } \\
& \mathrm{Li}_{k}(x)=\int_{2}^{x} \frac{d t}{\log ^{k} t} \quad \text { (definite integral). }
\end{aligned}
$$

Denote by li $x$ the conventional logarithmic integral (principal value):

$$
\operatorname{li} x=\int_{0}^{x} \frac{d t}{\log t}=\int_{2}^{x} \frac{d t}{\log t}+1.04516 \ldots
$$

In PARI/GP, an easy way to compute li $x$ is as follows: $\operatorname{li}(\mathrm{x})=\operatorname{real}(-\operatorname{eint} 1(-\log (\mathrm{x})))$.

The integrals $F_{k}(x)$ and $\mathrm{Li}_{k}(x)=F_{k}(x)-F_{k}(2)$ can also be expressed in terms of li $x$. Integration by parts gives

$$
\int \frac{d x}{\log x}=\frac{x}{\log x}+\frac{x}{\log ^{2} x}+\frac{2 x}{\log ^{3} x}+\frac{6 x}{\log ^{4} x}+\cdots+\frac{(k-2) ! x}{\log ^{k-1} x}+(k-1) ! \int \frac{d x}{\log ^{k} x} .
$$


Therefore,

$$
\begin{aligned}
& F_{2}(x)=\frac{1}{1 !}\left(\operatorname{li} x-\frac{x}{\log x}\right)+C \\
& F_{3}(x)=\frac{1}{2 !}\left(\operatorname{li} x-\frac{x}{\log ^{2} x}(\log x+1)\right)+C \\
& F_{4}(x)=\frac{1}{3 !}\left(\operatorname{li} x-\frac{x}{\log ^{3} x}\left(\log ^{2} x+\log x+2\right)\right)+C \\
& F_{5}(x)=\frac{1}{4 !}\left(\operatorname{li} x-\frac{x}{\log ^{4} x}\left(\log ^{3} x+\log ^{2} x+2 \log x+6\right)\right)+C \\
& F_{6}(x)=\frac{1}{5 !}\left(\operatorname{li} x-\frac{x}{\log ^{5} x}\left(\log ^{4} x+\log ^{3} x+2 \log ^{2} x+6 \log x+24\right)\right)+C,
\end{aligned}
$$

and, in general,

$$
F_{k+1}(x)=\frac{1}{k !}\left(\operatorname{li} x-\frac{x}{\log ^{k} x} \sum_{j=1}^{k}(k-j) ! \log ^{j-1} x\right)+C .
$$

Using these formulas we can compute $\operatorname{Li}_{k}(x)$ for approximating $\pi_{c}(x)$ (the prime counting function for sequence $\mathbb{P}_{c}$ ) in accordance with the $k$-tuple equidistribution conjecture (6):

$$
\pi_{c}(x) \approx \frac{C_{k, \mathcal{H}}}{\varphi_{k, \mathcal{H}}(q)} \operatorname{Li}_{k}(x)=\frac{C_{k, \mathcal{H}}}{\varphi_{k, \mathcal{H}}(q)}\left(F_{k}(x)-F_{k}(2)\right) .
$$

The values of $\mathrm{li} x$, and hence $\operatorname{Li}_{k}(x)$, can be calculated without (numerical) integration. For example, one can use the following rapidly converging series for li $x$, with $n$ ! in the denominator and $\log ^{n} x$ in the numerator (see [55], formulas 1.6.1.8-9):

$$
\operatorname{li} x=\log \log x+\sum_{n=1}^{\infty} \frac{\log ^{n} x}{n \cdot n !} \quad \text { for } x>1 .
$$

\section{References}

1. Sloane, N.J.A. (Ed.) The On-Line Encyclopedia of Integer Sequences. 2019. Available online: https:/ / oeis. org/ (accessed on 2 May 2019).

2. Berndt, B.C. Ramanujan's Notebooks, IV; Springer: New York, NY, USA, 1994.

3. Nicely, T.R. First Occurrence Prime Gaps, Preprint. 2018. Available online: http://www.trnicely.net/gaps/ gaplist.html (accessed on 2 May 2019).

4. Cramér, H. On the order of magnitude of the difference between consecutive prime numbers. Acta Arith. 1936, 2, 23-46. [CrossRef]

5. Shanks, D. On maximal gaps between successive primes. Math. Comp. 1964, 18, 646-651. [CrossRef]

6. Granville, A. Harald Cramér and the distribution of prime numbers. Scand. Actuar. J. 1995, 1, 12-28. [CrossRef]

7. Baker, R.C.; Harman, G.; Pintz, J. The difference between consecutive primes, II. Proc. Lond. Math. Soc. 2001, 83, 532-562. [CrossRef]

8. Ford, K.; Green, B.; Konyagin, S.; Maynard, J.; Tao, T. Long gaps between primes. J. Am. Math. Soc. 2018, 31, 65-105. [CrossRef]

9. Funkhouser, S.; Ledoan, A.H.; Goldston, D.A. Distribution of large gaps between primes. arXiv 2018, arXiv:1802.07609.

10. Nicely, T.R.; Nyman, B. New prime gaps between $10^{15}$ and $5 \times 10^{16}$. J. Integer Seq. 2003, 6, 03.3.1.

11. Oliveira e Silva, T.; Herzog, S.; Pardi, S. Empirical verification of the even Goldbach conjecture and computation of prime gaps up to $4 \times 10^{18}$. Math. Comp. 2014, 83, 2033-2060. [CrossRef] 
12. Wolf, M. Some Conjectures on the Gaps between Consecutive Primes, Preprint. 1998. Available online: http:/ / www.researchgate.net/publication/2252793 (accessed on 2 May 2019).

13. Wolf, M. Some heuristics on the gaps between consecutive primes. arXiv 2011, arXiv:1102.0481.

14. Wolf, M. Nearest neighbor spacing distribution of prime numbers and quantum chaos. Phys. Rev. E 2014, 89, 022922. [CrossRef] [PubMed]

15. Cadwell, J.H. Large intervals between consecutive primes. Math. Comp. 1971, 25, 909-913. [CrossRef]

16. Kourbatov, A. Maximal gaps between prime k-tuples: A statistical approach. J. Integer Seq. 2013, 16, 13.5.2.

17. Kourbatov, A. Tables of record gaps between prime constellations. arXiv 2013, arXiv:1309.4053.

18. Ford, K. Large Gaps in Sets of Primes and Other Sequences. Presented at the School on Probability in Number Theory. Centre De Recherches Mathématiques, University of Montreal. 2018. Available online: http:/ /www. crm.umontreal.ca/2018/Nombres18/horaireecole/pdf/montreal_talk1.pdf (accessed on 2 May 2019).

19. Oliveira e Silva, T. Gaps between Twin Primes, Preprint. 2015. Available online: http://sweet.ua.pt/tos / twin_gaps.html (accessed on 2 May 2019).

20. Broughan, K.A.; Barnett, A.R. On the subsequence of primes having prime subscripts. J. Integer Seq. 2009, 12, 09.2.3.

21. Bayless, J.; Klyve, D.; Oliveira e Silva, T. New bounds and computations on prime-indexed primes. Integers 2013, 13, A43.

22. Batchko, R.G. A prime fractal and global quasi-self-similar structure in the distribution of prime-indexed primes. arXiv 2014, arXiv:1405.2900v2.

23. Guariglia, E. Primality, fractality and image analysis. Entropy 2019, 21, 304. [CrossRef]

24. Wolf, M. Some conjectures on primes of the form $m^{2}+1$. J. Comb. Number Theory 2013, 5, $103-131$.

25. Baker, R.C.; Zhao, L. Gaps between primes in Beatty sequences. Acta Arith. 2016, 172, 207-242. [CrossRef]

26. Mills, W.H. A prime-representing function. Bull. Am. Math. Soc. 1947, 53, 604. [CrossRef]

27. Salas, C. Base-3 repunit primes and the Cantor set. Gen. Math. 2011, 19, 103-107.

28. Fine, B.; Rosenberger, G. Number Theory. An Introduction via the Density of Primes; Birkhäuser: Cham, Switzerland, 2016.

29. Friedlander, J.B.; Goldston, D.A. Variance of distribution of primes in residue classes. Q. J. Math. Oxf. 1996, 47, 313-336. [CrossRef]

30. Hardy, G.H.; Littlewood, J.E. Some Problems of 'Partitio Numerorum.' III. On the Expression of a Number as a Sum of Primes. Acta Math. 1923, 44, 1-70. [CrossRef]

31. Bateman, P.T.; Horn, R.A. A heuristic asymptotic formula concerning the distribution of prime numbers. Math. Comp. 1962, 16, 363-367. [CrossRef]

32. Golubev, V.A. Generalization of the functions $\varphi(n)$ and $\pi(x)$. Časopis Pro Pěstování Matematiky 1953, 78, $47-48$.

33. Golubev, V.A. Sur certaines fonctions multiplicatives et le problème des jumeaux. Mathesis 1958, 67, 11-20.

34. Golubev, V.A. Exact formulas for the number of twin primes and other generalizations of the function $\pi(x)$. Časopis Pro Pěstování Matematiky 1962, 87, 296-305.

35. Sándor, J.; Crstici, B. Handbook of Number Theory II; Kluwer: Dordrecht, The Netherlands, 2004.

36. Alder, H.L. A generalization of the Euler phi-function. Am. Math. Mon. 1958, 65, 690-692.

37. Dirichlet, P.G.L. Beweis des Satzes, dass jede unbegrenzte arithmetische Progression, deren erstes Glied und Differenz ganze Zahlen ohne gemeinschaftlichen Factor sind, unendlich viele Primzahlen enthält. Abhandlungen der Königlichen Preußischen Akademie der Wissenschaften zu Berlin 1837, 48, 45-71.

38. Gumbel, E.J. Statistics of Extremes; Columbia University Press: New York, NY, USA, 1958.

39. Ares, S.; Castro, M. Hidden structure in the randomness of the prime number sequence? Physica A 2006, 360, 285-296. [CrossRef]

40. Goldston, D.A.; Ledoan, A.H. On the differences between consecutive prime numbers, I. In Proceedings of the Integers Conference 2011: Combinatorial Number Theory, Carrollton, GA, USA, 26-29 October 2011; pp. 37-44.

41. Kourbatov, A. On the $n$th record gap between primes in an arithmetic progression. Int. Math. Forum 2018, 13, 65-78. [CrossRef]

42. Krug, J. Records in a changing world. J. Stat. Mech. Theory Exp. 2007, 2007, P07001. [CrossRef]

43. Kourbatov, A. On the distribution of maximal gaps between primes in residue classes. arXiv 2016, arXiv:1610.03340. 
44. Brent, R.P. Twin primes (seem to be) more random than primes. Presented at the Second Number Theory Down Under Conference, Newcastle, Australia, 24-25 October 2014. Available online: http:/ / maths-people. anu.edu.au/ brent/pd/twin_primes_and_primes.pdf (accessed on 2 May 2019).

45. Pintz, J. Cramér vs Cramér: On Cramér's probabilistic model for primes. Functiones Approximatio 2007, 37, 361-376. [CrossRef]

46. Kourbatov, A. The distribution of maximal prime gaps in Cramér's probabilistic model of primes. Int. J. Stat. Probab. 2014, 3, 18-29. [CrossRef]

47. Li, J.; Pratt, K.; Shakan, G. A lower bound for the least prime in an arithmetic progression. Q. J. Math. 2017, 68, 729-758.

48. Wolf, M. First Occurrence of a Given Gap Between Consecutive Primes, Preprint. 1997. Available online: http:/ / citeseerx.ist.psu.edu/viewdoc/download?doi=10.1.1.52.5981\&rep=rep1\&type=pdf (accessed on 2 May 2019).

49. Sun, Z.-W. Conjectures involving arithmetical sequences. In Number Theory: Arithmetic in Shangri-La, Proceedings of the 6th China-Japan Seminar, Shanghai, China, 15-17 August 2011; Kanemitsu, S., Li, H., Liu, J., Eds.; World Sci.: Singapore, 2013; pp. 244-258.

50. Kourbatov, A. Upper bounds for prime gaps related to Firoozbakht's conjecture. J. Integer Seq. 2015, 18, 15.11.2.

51. MathWave Technologies. EasyFit-Distribution Fitting Software. 2013. Available online: http://www. mathwave.com/easyfit-distribution-fitting.html (accessed on 2 May 2019).

52. Riesel, H. Prime Numbers and Computer Methods for Factorization; Birkhäuser: Boston, MA, USA, 1994.

53. Forbes, A.D. Prime k-Tuplets, Preprint. 2018. Available online: http:/ / anthony.d.forbes.googlepages.com/ ktuplets.htm (accessed on 2 May 2019).

54. Finch, S.R. Mathematical Constants; Cambridge University Press: Cambridge, UK, 2003.

55. Prudnikov, A.; Brychkov, Y.; Marichev, O. Integrals and Series. Vol. 1: Elementary Functions; Gordon and Breach: New York, NY, USA, 1986.

(C) 2019 by the authors. Licensee MDPI, Basel, Switzerland. This article is an open access article distributed under the terms and conditions of the Creative Commons Attribution (CC BY) license (http:/ / creativecommons.org/licenses/by/4.0/). 